\title{
Swarming on Random Graphs II
}

\author{
James H. von Brecht* ${ }^{*} \quad$ Benny Sudakov ${ }^{\dagger} \quad$ Andrea L. Bertozzi ${ }^{\ddagger}$
}

October 20, 2013

\begin{abstract}
We consider an individual-based model where agents interact over a random network via firstorder dynamics that involve both attraction and repulsion. In the case of all-to-all coupling of agents in $\mathbb{R}^{d}$ this system has a lowest energy state in which an equal number of agents occupy the vertices of the $d$-dimensional simplex. The purpose of this paper is to study the behavior of this model when the interaction between the $N$ agents occurs according to an Erdös-Rényi random graph $\mathcal{G}(N, p)$ instead of all-to-all coupling. In particular, we study the effect of randomness on the stability of these simplicial solutions, and provide rigorous results to demonstrate that stability of these solutions persists for probabilities greater than $N p=O(\log N)$. In other words, only a relatively small number of interactions are required to maintain stability of the state. The results rely on basic probability arguments together with spectral properties of random graphs.
\end{abstract}

\section{Introduction}

Individual-based models (IBM) have proven exceedingly useful for reproducing a wide variety of collective behaviors. Each individual in an IBM defines a "particle" that typically interacts with all other particles according to a specified potential function. The potential $V(s)$ and the interaction kernel $g(s):=-V^{\prime}(s)$ encode the precise dependence of the interaction on inter-particle distance, or the distance between individuals, and therefore widely vary between applications and across disciplines. The mathematics of such particle systems pervades many disciplines: it appears in models that range from physics, chemistry and biology to control theory and engineering. Classical examples from physics and chemistry include the distribution of electrons in the Thomson problem [44, 34, 2, 50, 8, 9, 25] and VSEPR theory. More modern applications in these areas include protein folding [33, 42], colloid stability [46, 47, 22] and the self-assembly of nanoparticles into supramolecular structures $[17,20,16,53]$. In biology, similar mathematical models help explain the complex phenomena observed in flocking [29, 10, 48], viral capsids [18, 52], locust swarms [12, 5] and colonies of bacteria $[45,11]$ or ants [4]. In engineering, non-local particle models have been successfully used in many areas of cooperative control [51, 23], including applications to robotic swarming [7, 51, 15, 14].

In each of these disciplines, the most pervasive component of such models consists of the total contribution from all pairwise isotropic interactions between individuals in the particle group. A special case of such models consists of $N$ interacting individuals obeying first-order dynamics under a repulsive-attractive interaction. By repulsive-attractive, we mean that the interaction kernel $g(s)$ has a single root $g(R)=0$ and that $g(s)$ is positive for $s<R$ and negative otherwise. Under these choices the system of $N$ ordinary differential equations

$$
\frac{\mathrm{d} \mathbf{x}_{i}}{\mathrm{~d} t}=\sum_{j \neq i}^{N} g\left(\frac{1}{2}\left|\mathbf{x}_{i}-\mathbf{x}_{j}\right|^{2}\right)\left(\mathbf{x}_{i}-\mathbf{x}_{j}\right)
$$

governs the evolution of the particles in time.

\footnotetext{
*Department of Mathematics, UCLA, Los Angeles, CA 90095. Email: jub@math.ucla.edu

${ }^{\dagger}$ Department of Mathematics, ETH, 8092 Zurich, Switzerland and Department of Mathematics, UCLA, Los Angeles, CA 90095. Email: benjamin.sudakov@math.ethz.ch

${ }^{\ddagger}$ Department of Mathematics, UCLA, Los Angeles, CA 90095. Email: bertozzi@math.ucla.edu
} 
This model assumes an all-to-all interaction structure between individuals. In other words, each individual interacts with every other individual in the particle group. This assumption can prove unrealistic in engineered systems with a large number of particles. In robotics, for instance, all-to-all communication can prove prohibitively expensive for a large number of robots, and the all-to-all structure may break due to random communication failures between individuals as well. We therefore aim to understand how the collective behavior of the particle system (1) is affected by the presence of a random network structure between individuals. For simplicity we represent the random network as an Erdös-Rényi random graph that remains fixed throughout time. Thus given a number $N$ of individuals let $E=\left\{e_{i j}\right\}_{i, j=1}^{N}$ denote the adjacency matrix of an undirected random graph drawn from $\mathcal{G}(N, p)$ : for $j \geq i$ the $e_{i j} \sim B(1, p)$ denote independent Bernoulli random variables and $e_{j i}=e_{i j}$ otherwise on the lower triangle. The basic particle model then becomes

$$
\frac{\mathrm{d} \mathbf{x}_{i}}{\mathrm{~d} t}=\sum_{j \neq i}^{N} e_{i j} g\left(\frac{1}{2}\left|\mathbf{x}_{i}-\mathbf{x}_{j}\right|^{2}\right)\left(\mathbf{x}_{i}-\mathbf{x}_{j}\right)
$$

in order to incorporate the random communication network between individuals.

The purely linear case $g(s) \equiv 1$ of the system (2) appears in studies of consensus and synchronization algorithms on a random graph $[24,36,19,37]$. Related models also frequently demand a theoretical understanding how a (possibly dynamic) random network interaction structure affects well-understood, deterministic behaviors such as phase transitions [1], consensus and synchronization [35, 43], and the emergence of collective behavior in locust swarms [21]. In matrix form the linear version of system (2) reads

$$
\frac{\mathrm{d} \mathfrak{X}}{\mathrm{d} t}=-[L \otimes \mathrm{Id}] \mathfrak{X}
$$

where $\mathfrak{X}=\left(\mathbf{x}_{1}, \ldots, \mathbf{x}_{N}\right)$ is the vector of all individuals $\mathbf{x}_{i} \in \mathbb{R}^{d}, L$ is the graph-Laplacian matrix and $\otimes$ denotes the Kronecker product. By definition, consensus for this system occurs if $\left\|\mathbf{x}_{i}(t)-\mathbf{x}_{j}(t)\right\| \rightarrow 0$ as $t \rightarrow \infty$, and since the graph is undirected consensus will occur if and only if the graph is connected $[41,38,40,31,30,27,28,6,39]$. Note that the matrix $L \otimes \operatorname{Id}$ necessarily has a $d$-dimensional nullspace $\mathcal{N}_{d}$ spanned by "constant" vectors of the form $\mathfrak{X}=(\mathbf{v}, \ldots, \mathbf{v}) \in \mathbb{R}^{N d}$ where $\mathbf{v} \in \mathbb{R}^{d}$ is fixed. The emergence of consensus therefore occurs if and only if the stability condition

$$
\max _{\left\{\mathbf{v} \in \mathcal{N}_{d}^{+}:\|\mathbf{v}\|=1\right\}}-\langle\mathbf{v},[L \otimes \operatorname{Id}] \mathbf{v}\rangle<0
$$

holds. In this way spectral properties of systems of the form $L \otimes$ Id determines the long time behavior of differential equations. Our analysis of the nonlinear variant (2) proceeds similarly. We shall analyze the spectral properties of matrices of the form $L_{\tilde{\mathcal{G}}} \otimes M$, where $\tilde{\mathcal{G}}$ denotes a sub-graph of the interaction structure and $M \in \mathbb{M}_{d \times d}$ denotes a symmetric, deterministic and positive semi-definite matrix. A stability condition similar to (4) will then determine long term behavior of the random system (2).

Our motivation for studying random linear systems of this form has its origins in elementary dynamical systems theory. Specifically, we may analyze the stability of an equilibrium solution to a system of random, non-linear ordinary differential equations by linearizing the ODE system about the equilibrium. This linearization process allows us to apply well-developed techniques from random matrix theory to study properties of random differential equations. A linear stability analysis of the equilibria of (2) requires performing two tasks. The first task entails finding those configurations of individuals that lie in equilibrium, i.e.

$$
\sum_{j \neq i}^{N} e_{i j} g\left(\frac{1}{2}\left|\mathbf{x}_{i}-\mathbf{x}_{j}\right|^{2}\right)\left(\mathbf{x}_{i}-\mathbf{x}_{j}\right)=\mathbf{0} \in \mathbb{R}^{d}, \quad \forall 1 \leq i \leq N .
$$

The second task couples a linearization of (2) around the equilibrium together with an analysis of the eigenvalues of the resulting matrix. Even in the deterministic case with all-to-all coupling, that is when $p=1$ and $e_{i j} \equiv 1$ for all $(i, j)$, describing the equilibria of (2) can prove quite challenging. The introduction of randomness into the underlying interaction structure only adds further complications. While the symmetry of the interaction structure in the all-to-all case permits the description of equilibria by means of analytical formulae in some cases, the presence of any randomness whatsoever immediately breaks this symmetry. An 
analytical description of equilibria proves nearly impossible as a result. In other words, as soon as the edge probability $p<1$ the equilibria of the fully coupled system can destabilize immediately. This leads to the formation of some other complicated, random equilibrium configuration (see figure 1, top row). As a result, we cannot reduce the study of stability to a pure random matrix problem since we do not have an adequate description of the equilibrium itself.
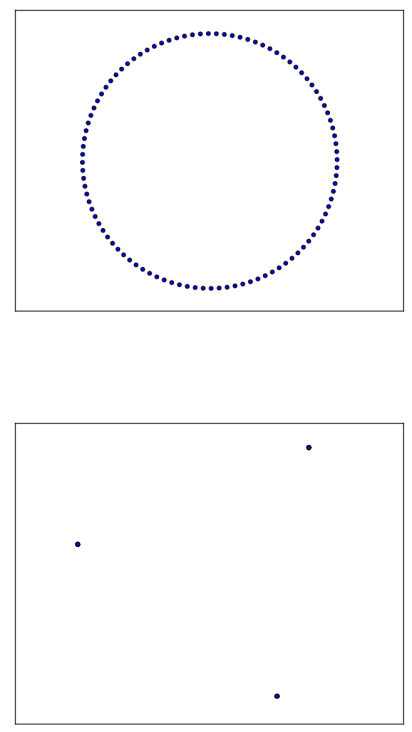

$p=1$
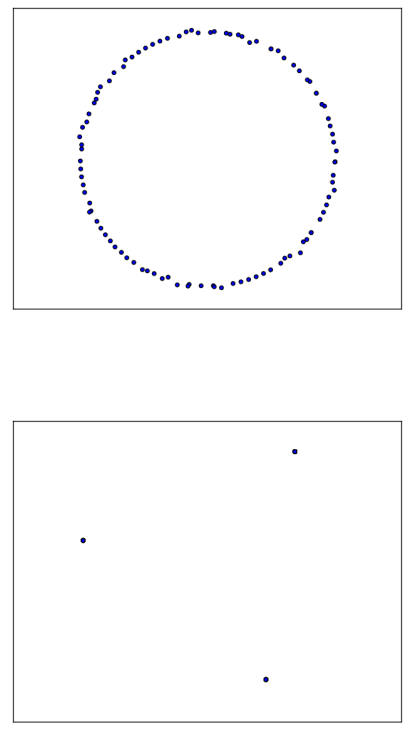

$p=.99$
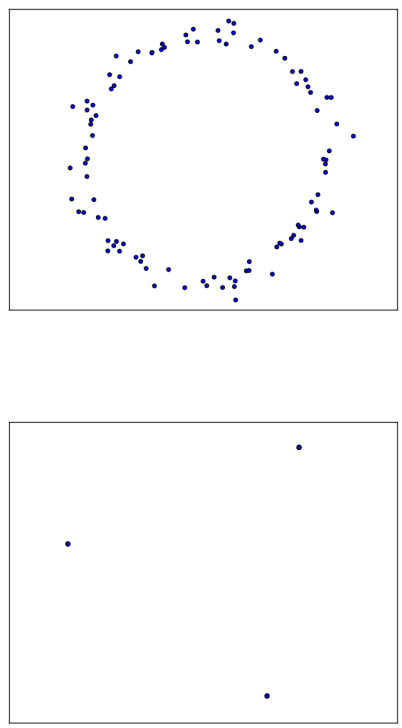

$p=.5$

Figure 1: Top row: A set of particles equally distributed along a ring defines an equilibrium under all-toall coupling. If $p<1$ the ring no longer defines an equilibrium and, as $p$ decreases, settle into a random equilibrium instead (final state shown). Bottom row: A simplex defines an equilibrium under all choices of graphs and also remains stable for relatively small edge probabilities $p$. Small initial perturbations (not shown) of the simplex decay and particles reoccupy the original simplex as $t \rightarrow \infty$ (final state shown).

To avoid this difficulty, i.e. the random, non-linear problem of finding equilibria of (2), we focus our efforts on a special class of equilibria to (2) that satisfy (5) under all possible realizations of the random graph. We must therefore allow each $e_{i j}$ to be zero or one arbitrarily. If we allow each $e_{i j}$ to take on the value zero or one arbitrarily, in order to not affect (5) then we must have

$$
g\left(\frac{1}{2}\left|\mathbf{x}_{i}-\mathbf{x}_{j}\right|^{2}\right)\left(\mathbf{x}_{i}-\mathbf{x}_{j}\right)=\mathbf{0}
$$

for any possible choice of distinct particle indices. As a consequence, for all $(i, j)$ either $\mathbf{x}_{i}=\mathbf{x}_{j}$ or $\left|\mathbf{x}_{i}-\mathbf{x}_{j}\right|=$ $\sqrt{2 R}$ where $R$ denotes the root of the interaction kernel. Each of the particles therefore lie at the vertices of a regular simplex in $\mathbb{R}^{d}$ whose edge length is determined by $R$. This restriction, i.e. that the particles lie in equilibrium regardless of their interaction structure, necessarily reduces our study to the class of so-called simplex configurations. These simplex configurations generalize the one-dimensional simplex equilibrium or "compromise solution" studied in [49], so named due to its similarity with the classical consensus-type algorithms in control theory. This is the particular choice of equilibrium where equal numbers $n=N / 2$ of particles occupy both vertices of the one dimensional simplex, so that $\mathbf{x}_{1}=\cdots=\mathbf{x}_{n}=0$ and $\mathbf{x}_{n+1}=$ $\cdots=\mathbf{x}_{N}=\sqrt{2 R}$ up to a reordering of the particle indices. Unlike the equilibria of $(2)$ that require the symmetry of all-to-all coupling, these simplex equilibria do not immediately destabilize with the introduction of randomness. Instead, they can remain stable even for relatively small values of $p$ (see figure 1 , bottom row). Moreover, the stability analysis of these equilibria reduces to a study of the eigenvalues of the matrix that results by linearizing (2) around the simplex configuration. In this manner, the stability analysis reduces to a pure random matrix problem. 
We refer to the one-dimensional system as a compromise model because the individuals in the each group (the two vertices of the $1 \mathrm{~d}$ simplex) prefer to remain at a fixed distance away from all other individuals; however, their attraction to the other group forces them to coexist at the same location with half of the total number of individuals in the group. The equilibrium therefore represents a compromise between these two competing effects. The associated linear system

$$
\kappa L_{1}-L_{2}
$$

also contrasts with the classical consensus case (3); the matrices $L_{1}$ and $L_{2}$ equal the graph Laplacians formed from two subgraphs of the full interaction structure. The first subgraph contains only those edges that do not connect the two groups and the second subgraph contains only those edges that do connect the two groups. We can therefore interpret the linear system as a competition between positive semi-definite Laplacian matrices. Our interest lies in determining when the stability condition (4) holds for the more general linear system (6). Our main result is a proof of the following theorem given in section 3, which settles a conjecture originally posed in [49]. We then demonstrate in section 4 how to extend the analysis in order to prove similar results for higher dimensional simplex configurations.

Main Result 1.1. Let $0<\kappa<1$ and let $e_{i j}$ denote the $N \times N$ adjacency matrix of an Erdös-Rényi random graph $\mathcal{G}(N, p)$. There exists a constant $p_{0 c}$ (independent of $N$ ) with the following property. If

$$
p \geq\left(p_{0 c}+\epsilon\right) \frac{\log N}{N}
$$

for some $\epsilon>0$ then the stability condition (4) holds for the system (6) asymptotically almost surely. If

$$
p \leq\left(p_{0 c}-\epsilon\right) \frac{\log N}{N}
$$

the stability condition (4) fails for the system (6) asymptotically almost surely. Moreover, $p_{0 c}$ depends on $\kappa$ through the relation

$$
p_{0 c}=\frac{2}{2-\kappa^{-\kappa /(\kappa+1)}(1+\kappa)} .
$$

\section{Preliminary Material}

We first pause to fix our notation, terminology and to collect a few preliminary lemmas before we proceed with our main results. Capital roman letters such as $A, B, C$ will always refer to matrices, while the corresponding lower-case letters $a_{i j}, b_{i j}, c_{i j}$ will denote the corresponding entries. We reserve Id for the identity matrix, $\mathbf{0}=(0, \ldots, 0)^{t}$ for the zero vector and $\mathbf{1}=(1, \ldots, 1)^{t}$ for the constant vector. We shall use $\mathbf{e}_{1}=(1,0,1,0, \ldots, 1,0)^{t}$ to denote the vector in $\mathbb{R}^{2 n}$ with $n$ copies of $(1,0)^{t}$ and use $\mathbf{e}_{2}$ denote the analogous vector with $(0,1)^{t}$ repeated $n$ times. The size of each of the preceeding is elucidated by the context in which it appears. For an $n \times n$ symmertic matrix $A$, we let $\lambda_{i}(A)$ denote its $i^{\text {th }}$ eigenvalue sorted in decreasing order. In other words, we have

$$
\lambda_{1}(A) \geq \lambda_{2}(A) \cdots \geq \lambda_{n}(A)
$$

where each eigenvalue appears according to its algebraic multiplicity.

Given a sequence of measurable events $\left\{W_{n}\right\}_{n=1}^{\infty}$, each of which lies in some (possibly different) probability space, we say that the sequence of events $\left\{W_{n}\right\}_{n \geq 1}$ holds asymptotically almost surely (a.a.s.) if

$$
\mathbb{P}\left(W_{n}\right) \rightarrow 1 \text { as } n \rightarrow \infty .
$$

Here and in what follows, $\mathbb{P}$ always denotes the measure on the probability space in which the relevant event lies. We denote by $B(1, p)$ a Bernoulli random variable with parameter $p$ and $B(n, p)$ the corresponding Binomial distribution. We use $\mathbb{E}(X)$ to denote the mean or expectation of the random variable $X$ while the notation $X \sim Y$ signifies that the random variables $X$ and $Y$ have the same distribution. We reserve $E=\left\{e_{i j}\right\}$ for the random matrix that corresponds to the adjacency matrix of Erdös-Rényi random graph. Given such a random graph on $n$ vertices with adjacency matrix $E$ and a symmetric, deterministic matrix 
$M \in \mathbb{M}_{d \times d}(\mathbb{R})$ we form the generalized adjacency matrix $A(M) \in \mathbb{M}_{n d \times n d}(\mathbb{R})$ generated by $M$ according to the formula

$$
A(M):=E \otimes M,
$$

where $A \otimes B$ denotes the Kronecker product of two matrices. These matrices naturally appear in the linearization of (2) around the family of simplex equlibria.

Our arguments rely on two types of probabilistic estimates that apply either to a sum of random variables or to a random matrix when a "mean-zero" hypothesis applies. Roughly speaking, these estimates allow us to reduce our analysis to the "mean" of these components. This "mean" is then usually much easier to analyze than the full component itself. Lemma 2.1 below, which states a variant of the well-known Chernoff bound (c.f. [26]), provides the first result of this type. It furnishes tail estimates on a sum of random variables $X_{i}$ that satisfy the bona-fide mean zero hypothesis $\mathbb{E}\left(X_{i}\right)=0$ -

Lemma 2.1. (Chernoff Bound) Let $X_{1}, \ldots, X_{m}$ denote discrete, independent random variables satisfying $\mathbb{E}\left(X_{i}\right)=0$ and $\left|X_{i}\right| \leq 1$. If $\mathbb{E}\left(X_{i}^{2}\right)=\sigma_{i}^{2}$ and $\sigma^{2} \geq \sum \sigma_{i}^{2}$, then for any $0 \leq \lambda \leq 2 \sigma$

$$
\mathbb{P}\left(\left|\sum_{i=1}^{m} X_{i}\right| \geq \lambda \sigma\right) \leq 2 \mathrm{e}^{-\lambda^{2} / 4} .
$$

When dealing with generalized adjacency matrices of the form (31), it proves natural to decompose a given a vector $\mathbf{x} \in \mathbb{R}^{n d}$ as $\mathbf{x}=\left(\mathbf{x}_{1}, \ldots, \mathbf{x}_{n}\right)^{t}$, where each $\mathbf{x}_{i} \in \mathbb{R}^{d}$. The "mean-zero" hypothesis in this context enforces orthogonality of $\mathbf{x} \in \mathbb{R}^{n d}$ with respect to the "constant vectors" $\mathbf{v}_{c}=(\mathbf{w}, \ldots, \mathbf{w})^{t} \in \mathbb{R}^{n d}$, or in other words it holds that

$$
\sum_{i=1}^{n} \mathbf{x}_{i}=\mathbf{0}
$$

If we denote the corresponding subset of the unit ball $\mathcal{S}^{\text {nd }} \subset \mathbb{R}^{n d}$ as

$$
\mathcal{S}_{0}^{n d}:=\left\{\mathrm{x}: \sum_{i} \mathbf{x}_{i}=\mathbf{0}, \quad \sum_{i}\left\|\mathbf{x}_{i}\right\|_{2}^{2} \leq 1\right\},
$$

then we may state the second type of probabilistic estimate as follows -

Theorem 2.2. Let $\alpha$ and $c_{0}$ denote arbitrary positive constants. Let $E$ denote the adjacency matrix of a random graph from $\mathcal{G}(n, p), M \in \mathbb{M}_{d \times d}(\mathbb{R})$ denote an arbitrary symmetric matrix and $A(M)=E \otimes M$ the corresponding generalized adjacency matrix. If $n p>c_{0} \log n$ then there exists a constant $c=c\left(\alpha, c_{0}, d,\|M\|_{2}\right)>0$ so that the estimate

$$
\max _{(\mathbf{x}, \mathbf{y}) \in \mathcal{S}_{0}^{n d} \times \mathcal{S}^{n d}}|\langle\mathbf{x}, A \mathbf{y}\rangle| \leq c \sqrt{n p}
$$

holds with probability at least $1-n^{-\alpha}$.

This theorem represents a generalization (and slight improvement when $d=1$ ) of a theorem from [13], and its proof follows that of [13] closely. We include it for completeness in the appendix.

The reductions that these estimates permit allow us to focus our efforts on the "mean" of the random matrix under consideration. This "mean" essentially consists of weighted differences between independent binomial distributions. Our method of estimating these weighted differences requires a few standard facts regarding special functions, namely the gamma function $\Gamma(z)$ and the digamma function $\Psi^{0}(z)$ defined by

$$
\Psi^{0}(z):=\frac{\mathrm{d}}{\mathrm{d} z} \log \Gamma(z)
$$

For the gamma function, we shall use Stirling's formula both in terms of upper and lower bounds

$$
1 \leq \frac{\Gamma(z+1)}{\sqrt{2 \pi z}(z / \mathrm{e})^{z}} \leq \frac{\mathrm{e}}{\sqrt{2 \pi}} \text { for } z \in \mathbb{N}
$$


and in terms of the asymptotic relation for $z \in \mathbb{R}^{+}$

$$
\log \Gamma(z+1)=\left(z+\frac{1}{2}\right) \log z-z+O(1) \quad \text { as } \quad z \rightarrow \infty .
$$

For the digamma function $\Psi^{0}(z)$ we shall use the properties (c.f. [3], [32])

$$
\begin{gathered}
\Psi^{0}(z) \quad \text { is increasing for } z>0 \\
\Psi^{0}(z+1)=\log z+O\left(\frac{1}{z}\right) \quad \text { as } \quad z \rightarrow \infty \\
\log z \leq \Psi^{0}(z+1) \leq \log (z+1) .
\end{gathered}
$$

With these preliminaries in place, we may now proceed to formalize and prove our main results. The next section formalizes the linear stability problem for one-dimensional simplex equilibria and also proves a sharp threshold for when stability of these solutions holds asymptotically almost surely. That is, it resolves conjecture 1.1. Section 4 extends this formalization and analysis to the higher dimensional case.

\section{Problem Statement in One Dimension}

In this section we shall first describe in greater detail the random stability matrix that results from linearizing (2) around the one-dimensional simplex equilibrium, or the "compromise" solution. We then proceed with a few preliminary reductions that allow us to determine stability or instability of this solution by analyzing a random, diagonal matrix instead of the full stability matrix. We then fully characterize the threshold...etc.

To begin, recall that we obtain the one-dimensional simplex equilibrium by subdividing a group of $N=2 n$ scalar particles $\mathbf{x}_{i} \in \mathbb{R}$ into two equal-sized groups of $n$ individuals then placing them a distance $R$ apart:

$$
\mathbf{x}_{1}=\cdots=\mathbf{x}_{n}=0 \quad \text { and } \quad \mathbf{x}_{n+1}=\cdots=\mathbf{x}_{N}=R .
$$

As always let $E \in \mathbb{M}_{N \times N}(\mathbb{R})$ denote the adjacency matrix of the $\mathcal{G}(N, p)$ random graph that determines the interaction structure. We partition the adjacency matrix as

$$
E=\left(\begin{array}{cc}
A & B \\
B^{t} & C
\end{array}\right), \quad A=A^{t}, \quad C=C^{t}, \quad A, B, C \in M_{n \times n}(\mathbb{R})
$$

where $A$ and $C$ correspond to intra-group edges (interactions within the same group) and $B$ corresponds to inter-group edges (interactions across groups). The resulting stability matrix $L$ for the compromise equilibrium reads

$$
L=\kappa L_{1}-L_{2}, \quad L_{1}=\left(\begin{array}{cc}
D_{A}-A & 0 \\
0 & D_{C}-C
\end{array}\right), \quad L_{2}=\left(\begin{array}{cc}
D_{B} & -B \\
-B^{t} & D_{B^{t}}
\end{array}\right) .
$$

For each $M \in\left\{A, B, B^{t}, C\right\}$ the diagonal matrix $D_{M}$ has non-zero elements corresponding to row sums of $M$, so that $L_{1}$ and $L_{2}$ correspond to positive semi-definite graph Laplacian matrices. Here $0<\kappa<1$ is a system parameter fully determined by $g(s)$, its first derivative and the distance $R$ between the two compromised groups.

The characterization of stability or instability relies on determining when the eigenvalues of $L$ have the appropriate sign. As 1 always defines an eigenvector with eigenvalue zero, our interest lies in placing probabilistic bounds on when $\lambda_{1}(L)=0$ and the second-largest eigenvalue $\lambda_{2}(L)$ of $L$ is non-positive asymptotically almost surely. This is a necessary condition for stability of the compromise model. We therefore aim to establish conditions on $p$ for when the stability condition

$$
\max _{\left\{\mathbf{v} \in 1^{\perp}:\|\mathbf{v}\|=1\right\}}\langle\mathbf{v}, L \mathbf{v}\rangle<0
$$

holds asymptotically almost surely. The following subsections rigorously establish a critical threshold $p=p_{c}$ for this stability condition to hold. 


\subsection{Reduction to the Diagonal Component}

Clearly, if $L$ has a positive diagonal entry then $\lambda_{1}(L)>0$ and $\lambda_{2}(L) \geq 0$, so we may reduce to the case when the diagonal component $D$ of the stability matrix $L$

$$
D:=\left(\begin{array}{cc}
\kappa D_{A}-D_{B} & 0 \\
0 & \kappa D_{C}-D_{B^{t}}
\end{array}\right)
$$

has non-positive entries. The following lemma asserts that having $\lambda_{1}(D) \leq-c_{1} N p$ for some $c_{1}>0$ asymptotically almost surely suffices to guarantee that $\lambda_{1}(L)=0$ and $\lambda_{2}(L)<0$ asymptotically almost surely as well.

Lemma 3.1. Assume that $p=o(1)$, there exists a $c_{0}>0$ so that $N p \geq c_{0} \log N$ and a $c_{1}>0$ so that the diagonal component $(20)$ of $L$ satisfies $\lambda_{1}(D) \leq-c_{1} N p$ asymptotically almost surely. Then $\lambda_{2}(L)<0$ asymptotically almost surely.

Proof. Let $V$ denote the subspace of $\mathbb{R}^{N}$ that consists of mean-zero vectors, i.e. $V:=\left\{\mathbf{v} \in \mathbb{R}^{N}: \sum_{i} v_{i}=0\right\}$, and define $\mathbf{1}_{0} \in V$ as

$$
\mathbf{1}_{0}=\frac{1}{\sqrt{N}}\left(\begin{array}{r}
1 \\
-1
\end{array}\right)
$$

for $\mathbf{1} \in \mathbb{R}^{n}$, and note that $\left\|\mathbf{1}_{0}\right\|_{2}=1$. Let $\mathbf{v} \in V$ satisfy $\|\mathbf{v}\|_{2}=1$, and note that $\mathbf{v}$ decomposes as

$$
\mathbf{v}=\alpha \mathbf{1}_{0}+\beta \mathbf{y} \quad \text { for } \quad \mathbf{y} \in V_{0}^{\perp}:=\left\{\mathbf{v} \in \mathbb{R}^{N}: \sum_{i=1}^{n} v_{i}=\sum_{i=n+1}^{N} v_{i}=0\right\}
$$

where $\|\mathbf{y}\|_{2}=1$ and $\alpha^{2}+\beta^{2}=1$. The definition of $L_{1}$ implies $L_{1} \mathbf{1}_{0}=\mathbf{0}$, so that a direct computation of $\langle\mathbf{v}, L \mathbf{v}\rangle$ shows that

$$
\begin{aligned}
\langle\mathbf{v}, L \mathbf{v}\rangle & =\beta^{2}\langle\mathbf{y}, L \mathbf{y}\rangle-\alpha^{2}\left\langle\mathbf{1}_{0}, L_{2} \mathbf{1}_{0}\right\rangle-2 \alpha \beta\left\langle\mathbf{1}_{0}, L_{2} \mathbf{y}\right\rangle \\
& =\beta^{2}\langle\mathbf{y}, L \mathbf{y}\rangle-\frac{4 \alpha^{2}}{N} \sum_{i, j=1}^{n} b_{i j}-2 \alpha \beta\left\langle\mathbf{y}, L_{2} \mathbf{1}_{0}\right\rangle
\end{aligned}
$$

Define the random variables $X:=\sum_{i, j=1}^{n} b_{i j}$ and $Y:=\left\langle\mathbf{y}, L_{2} \mathbf{1}_{0}\right\rangle$. As $\mathbb{E}\left(b_{i j}\right)=p$ and the $b_{i j}$ are independent, it follows that $\mathbb{E}(X)=n^{2} p$ and $\operatorname{Var}(X)=n^{2} p(1-p)$. The Chernoff bound (c.f. lemma 2.1) then implies that

$$
\mathbb{P}(|X-\mathbb{E}(X)| \geq n \sqrt{n p(1-p)}) \leq 2 \mathrm{e}^{-n / 4}
$$

for all $n$ sufficiently large, so in particular it holds that $X / N=n p / 2+O(\sqrt{N p})$ asymptotically almost surely.

To estimate $Y$, write $\mathbf{y} \in V_{0}^{\perp}$ as $\mathbf{y}=\left(\mathbf{y}_{1}, \mathbf{y}_{2}\right)^{t}$ for $\mathbf{y}_{i} \in \mathbb{R}^{n}$, then recall that the definition of $L_{2}=L_{2}^{t}$ implies

$$
Y:=\left\langle L_{2} \mathbf{y}, \mathbf{1}_{0}\right\rangle=\frac{1}{\sqrt{N}}\left(\left\langle\mathbf{1}, D_{B} \mathbf{y}_{1}\right\rangle-\left\langle\mathbf{1}, B \mathbf{y}_{2}\right\rangle-\left\langle\mathbf{1}, D_{B^{t}} \mathbf{y}_{2}\right\rangle+\left\langle\mathbf{1}, B^{t} \mathbf{y}_{1}\right\rangle\right)
$$

It follows by definition that $D_{B} \mathbf{1}=B \mathbf{1}$ and $D_{B^{t}} \mathbf{1}=B^{t} \mathbf{1}$, which then implies

$$
Y=\frac{2}{\sqrt{N}}\left(\left\langle\mathbf{1}, B^{t} \mathbf{y}_{1}\right\rangle-\left\langle\mathbf{1}, B \mathbf{y}_{2}\right\rangle\right)=2\left\langle\mathbf{1}_{0}, E \mathbf{y}\right\rangle-\frac{2}{\sqrt{N}}\left(\left\langle\mathbf{1}, A \mathbf{y}_{1}\right\rangle-\left\langle\mathbf{1}, C \mathbf{y}_{2}\right\rangle\right) .
$$

Applying theorem 2.2 with $d=1$ shows that $\left\langle\mathbf{1}_{0}, E \mathbf{y}\right\rangle=O(\sqrt{N p})$ asymptotically almost surely. Define $\tilde{\mathbf{y}}_{1}:=\left(\mathbf{y}_{1}, \mathbf{0}\right)^{t}$ and $\tilde{\mathbf{1}}:=(\mathbf{1}, \mathbf{0})^{t}$, so that the equality $\left\langle\mathbf{1}, A \mathbf{y}_{1}\right\rangle=\left\langle\tilde{\mathbf{y}}_{1}, E \tilde{\mathbf{1}}\right\rangle$ holds. As $\left(\tilde{\mathbf{y}}_{1}, \mathbf{0}\right)^{t} \in \mathcal{S}_{0}^{N}$ and $E \sim \mathcal{G}(N, p)$, a direct application of theorem 2.2 with $d=1$ suffices to yield $\left|\left\langle\mathbf{1}, A \mathbf{y}_{1}\right\rangle\right|=\sqrt{n} O(\sqrt{n p})$ asymptotically almost surely. A similar argument demonstrates $\left|\left\langle\mathbf{1}, C \mathbf{y}_{2}\right\rangle\right|=\sqrt{n} O(\sqrt{n p})$ asymptotically almost surely as well. Thus $|Y|=O(\sqrt{n p})$ asymptotically almost surely. That $2 \alpha \beta \leq \alpha^{2}+\beta^{2}=1$ then implies

$$
\langle\mathbf{v}, L \mathbf{v}\rangle \leq \beta^{2}\langle\mathbf{y}, L \mathbf{y}\rangle-\frac{4 \alpha^{2}}{N} X-2 \alpha \beta Y \leq-\alpha^{2} N p+\beta^{2}\langle\mathbf{y}, L \mathbf{y}\rangle+O(\sqrt{N p})
$$


asymptotically almost surely.

It remains to estimate $\langle\mathbf{y}, L \mathbf{y}\rangle$. Again write $\mathbf{y} \in V_{0}^{\perp}$ as $\mathbf{y}=\left(\mathbf{y}_{1}, \mathbf{y}_{2}\right)^{t}$ for $\mathbf{y}_{i} \in \mathbb{R}^{n}$, and recall that $\left\|\mathbf{y}_{i}\right\|_{2} \leq 1$ and $\mathbf{1} \cdot \mathbf{y}_{i}=0$ by definition. Thus

$$
\beta^{2}\langle\mathbf{y}, L \mathbf{y}\rangle=\beta^{2}\langle\mathbf{y}, D \mathbf{y}\rangle-\beta^{2}(1+\kappa)\left(\left\langle\mathbf{y}_{1}, A \mathbf{y}_{1}\right\rangle+\left\langle\mathbf{y}_{2}, B \mathbf{y}_{2}\right\rangle\right)+\beta^{2}\langle\mathbf{y}, E \mathbf{y}\rangle .
$$

As each of $\mathbf{y}_{1}, \mathbf{y}_{2}$ and $\mathbf{y}$ have zero mean and $\|\mathbf{y}\|_{2}=1$, it follows from theorem 2.2 that

$$
\beta^{2}\langle\mathbf{y}, L \mathbf{y}\rangle=\beta^{2}\langle\mathbf{y}, D \mathbf{y}\rangle+O(\sqrt{N p}) \leq \lambda_{1}(D)\|\mathbf{y}\|_{2}^{2} \beta^{2}+O(\sqrt{N p}) \leq-c_{1} N p \beta^{2}+O(\sqrt{N p}) .
$$

As a consequence, that $\alpha^{2}+\beta^{2}=1$ then implies

$$
\max _{\mathbf{v} \in V:\|\mathbf{v}\|_{2}=1}\langle\mathbf{v}, L \mathbf{v}\rangle \leq-\min \left\{1, c_{1}\right\} N p+O(\sqrt{N p})<0
$$

asymptotically almost surely. Noting that $\mathbf{1}$ always defines an eigenvector of $L$ then yields the desired result.

\subsection{Estimating the Diagonal}

With this reduction in hand, we may now proceed with the task of establishing the hypothesis $\lambda_{1}(D) \leq$ $-c_{1} N p$ in the preceeding lemma. Each non-zero entry $D_{i i}$ of $D$ has exactly the same distribution, i.e. a difference of two independent binomial distributions (although dependencies exist between the diagonal entires themselves due to the undirected graph). Specifically, we have

$$
d_{i i} \sim \kappa X-Y
$$

for $X, Y \sim B(n, p)$ with $X$ and $Y$ independent. We therefore wish to estimate when $\mathbb{P}\left(d_{11} \geq-c_{1} N p\right)$ holds with probability sufficiently small to apply the union bound over all diagonal entries. In crude terms, for $N p=\Theta(\log n)$ and $N=2 n$ we have that

$$
\mathbb{P}\left(d_{11} \geq-c_{1} N p\right) \approx c_{2}(n) \mathrm{e}^{-c_{0}\left(\kappa, c_{1}\right) n p}, \quad c_{0}\left(\kappa, c_{1}\right)=c_{0}(\kappa, 0)+o(1) \quad\left(\text { as } c_{1} \rightarrow 0\right)
$$

for some function $c_{0}\left(\kappa, c_{1}\right)$ that depends on $\kappa$ and $c_{1}$ and some function $c_{2}(n)$ that grows (or decays) more slowly in $n$ than any power. A threshold therefore occurs when $c_{0}(\kappa, 0) n p=\log n$, or in other words when

$$
\mathbb{P}\left(d_{11} \geq 0\right) \approx c_{2}(n) \Theta\left(\frac{1}{n}\right)
$$

Indeed, if $c_{0}(\kappa, 0) n p \geq(1+\epsilon) \log n$ then $\mathbb{P}\left(d_{11}>-c_{1} N p\right) \approx n^{-(1+\epsilon)}$ and we may apply the union bound over all $2 n$ diagonal entries. On the other hand, if $c_{0}(\kappa, 0) n p \leq(1-\epsilon) \log n$ then $\mathbb{P}\left(d_{11} \geq c_{1} N p\right) \approx n^{-(1-\epsilon)}$ and the union bound fails. In this case, we expect $L$ to have positive diagonal entries and therefore instability to occur.

To realize this program, we must have a method for calculating $c_{0}\left(\kappa, c_{1}\right)$ itself. Given $X \sim B(n, p)$ and $Y \sim B(n, p)$, let $Z=\kappa X-Y$ then define

$$
f_{n}\left(p, \kappa, c_{1}\right):=\mathbb{P}\left(Z \geq c_{1} n p\right)=\sum_{i=0}^{n}\left(\begin{array}{c}
n \\
i
\end{array}\right) p^{i}(1-p)^{n-i} \sum_{j=0}^{\left\lfloor\kappa i-c_{1} n p\right\rfloor}\left(\begin{array}{c}
n \\
j
\end{array}\right) p^{j}(1-p)^{n-j} .
$$

As the following lemmas demonstrate, we can estimate $f_{n}\left(p, \kappa, c_{1}\right)$ to the precision needed by considering only the largest term in the sum. Finding and estimating this term only involves calculus and a few properties of special functions.

Lemma 3.2. Suppose that there exists $\epsilon>0$ so that

$$
(1-\epsilon) \frac{\log n}{n} \leq p \leq(1-\epsilon) c_{0}(\kappa) \frac{\log n}{n}, \quad c_{0}(\kappa):=\frac{1}{2-(1+\kappa) \kappa^{-\frac{\kappa}{1+\kappa}}} .
$$

If $0<c_{1}<\kappa^{\frac{1}{1+\kappa}}$ is sufficiently small, depending only on $(\epsilon, \kappa)$, then there exists a universal constant $c^{\prime}>0$ so that

$$
f_{n}\left(p, \kappa, c_{1}\right) \geq c^{\prime} n^{-1+\epsilon / 2} .
$$


Proof. For a fixed $n>0$, define

$$
\begin{array}{lll}
i_{0}:=\left\lceil\kappa^{-\frac{\kappa}{1+\kappa}} n p\right\rceil=\delta(n) n p, & \delta(n)=\kappa^{-\frac{\kappa}{1+\kappa}}\left(1+\epsilon_{1}\right), & \epsilon_{1}=O\left(\frac{1}{\log n}\right), \\
j_{0}:=\left\lfloor\left(1-c_{1} \kappa^{-\frac{1}{1+\kappa}}\right) \kappa i_{0}\right\rfloor=\gamma(n) n p, & \gamma(n)=\left(1-c_{1} \kappa^{-\frac{1}{1+\kappa}}\right) \kappa^{\frac{1}{1+\kappa}}\left(1+\epsilon_{2}\right), & \epsilon_{2}=O\left(\frac{1}{\log n}\right) .
\end{array}
$$

Write

$$
\left(\begin{array}{c}
n \\
i
\end{array}\right) p^{i}(1-p)^{n-i}=\mathrm{e}^{\Phi(i)} \quad \Phi(i):=\log n !-\log i !-\log (n-i) !+i \log \frac{p}{1-p}+n \log (1-p),
$$

and note that $f_{n}\left(p, \kappa, c_{1}\right)>\mathrm{e}^{\Phi\left(i_{0}\right)} \mathrm{e}^{\Phi\left(j_{0}\right)}$ since the pair $\left(i_{0}, j_{0}\right)$ contributes a singleton term in the sum. Indeed, as

$$
\left(1-c_{1} \kappa^{-\frac{1}{1+\kappa}}\right) \kappa i_{0} \leq \kappa i_{0}-c_{1} n p
$$

it follows that $j_{0}=\left\lfloor\left(1-c_{1} \kappa^{-\frac{1}{1+\kappa}}\right) \kappa i_{0}\right\rfloor \leq\left\lfloor\kappa i_{0}-c_{1} n p\right\rfloor$. Stirling's formula (15) for the factorial and the fact that $\log (1-p)=-p+O\left(p^{2}\right)$ together imply that

$$
\begin{aligned}
\Phi\left(i_{0}\right) & =n \log \frac{n}{n-i_{0}}-i_{0} \log \frac{i_{0}}{n-i_{0}}+\frac{1}{2} \log \frac{n}{i_{0}\left(n-i_{0}\right)}+i_{0} \log \frac{p}{1-p}+n \log (1-p)+O(1) \\
& =i_{0}-i_{0} \log \frac{i_{0}}{n-i_{0}}-\frac{1}{2} \log n p+i_{0} \log \frac{p}{1-p}-n p+O(1), \\
\Phi\left(j_{0}\right) & =j_{0}-j_{0} \log \frac{j_{0}}{n-j_{0}}-\frac{1}{2} \log n p+j_{0} \log \frac{p}{1-p}-n p+O(1) .
\end{aligned}
$$

The definitions of $i_{0}$ and $j_{0}$ combine with these estimates to yield

$$
\begin{aligned}
\Phi\left(i_{0}\right)+\Phi\left(j_{0}\right) & =\left[(1+\kappa) \kappa^{-\frac{\kappa}{1+\kappa}}-\left(2+c_{1}\right)\right] n p-\log n p+i_{0} \log \frac{p\left(n-i_{0}\right)}{i_{0}(1-p)}+j_{0} \log \frac{p\left(n-j_{0}\right)}{j_{0}(1-p)}+O(1), \\
& =\left[(1+\kappa) \kappa^{-\frac{\kappa}{1+\kappa}}-\left(2+c_{1}\right)\right] n p-\log n p+n p\left[\delta(n) \log \frac{1}{\delta(n)}+\gamma(n) \log \frac{1}{\gamma(n)}\right]+O\left(n p^{2}\right)+O(1) .
\end{aligned}
$$

From the definitions of $\delta(n), \gamma(n)$, the fact $\epsilon_{i}=O\left(\log ^{-1} n\right)$ and the fact $\log \left(1+\epsilon_{i}\right)=O\left(\log ^{-1} n\right)$ it follows that

$$
\begin{gathered}
\delta(n) \log \frac{1}{\delta(n)}+\gamma(n) \log \frac{1}{\gamma(n)}=c_{1} \log \left(\kappa^{\frac{1}{1+\kappa}}-c_{1}\right)-\kappa^{\frac{1}{1+\kappa}} \log \left(1-c_{1} \kappa^{-\frac{1}{1+\kappa}}\right)+O\left(\frac{1}{\log n}\right), \\
n p\left[\delta(n) \log \frac{1}{\delta(n)}+\gamma(n) \log \frac{1}{\gamma(n)}\right]=n p\left[c_{1} \log \left(\kappa^{\frac{1}{1+\kappa}}-c_{1}\right)-\kappa^{\frac{1}{1+\kappa}} \log \left(1-c_{1} \kappa^{-\frac{1}{1+\kappa}}\right)\right]+O(1) .
\end{gathered}
$$

As a consequence,

$$
\begin{aligned}
\Phi\left(i_{0}\right)+\Phi\left(j_{0}\right) & =\left[(1+\kappa) \kappa^{-\frac{\kappa}{1+\kappa}}-2\right] n p+h\left(c_{1}\right) n p-\log n p+O(1), \\
h\left(c_{1}\right) & :=\left[c_{1} \log \left(\kappa^{\frac{1}{1+\kappa}}-c_{1}\right)-\kappa^{\frac{1}{1+\kappa}} \log \left(1-c_{1} \kappa^{-\frac{1}{1+\kappa}}\right)-c_{1}\right] .
\end{aligned}
$$

By (22), this implies

$$
\Phi\left(i_{0}\right)+\Phi\left(j_{0}\right) \geq(\epsilon-1) \log n+h\left(c_{1}\right) n p-\log n p+O(1) \geq\left(\epsilon-1-\left|h\left(c_{1}\right)\right| c_{0}(\kappa)\right) \log n-\log n p+O(1) .
$$

As $h\left(c_{1}\right) \rightarrow 0$ as $c_{1} \rightarrow 0$, it follows that that $f_{n}\left(p, \kappa, c_{1}\right) \geq \exp \left(\Phi\left(i_{0}\right)+\Phi\left(j_{0}\right)\right) \geq n^{\epsilon / 2-1}$ for all $n$ sufficiently large if $c_{1}$ is sufficiently small, depending only on $\epsilon$ and $\kappa$, as claimed.

For $c_{1}>0$, define

$$
f_{n}\left(p, \kappa, c_{1}\right):=\mathbb{P}\left(Z \geq-c_{1} n p\right)=\sum_{i=0}^{n}\left(\begin{array}{c}
n \\
i
\end{array}\right) p^{i}(1-p)^{n-i} \sum_{j=0}^{\left\lfloor\kappa i+c_{1} n p\right\rfloor}\left(\begin{array}{c}
n \\
j
\end{array}\right) p^{j}(1-p)^{n-j} .
$$


Lemma 3.3. Suppose that there exist $c, \epsilon>0$ so that

$$
n p \geq(1+\epsilon) c_{0}(\kappa) \log n, \quad c_{0}(\kappa):=\frac{1}{2-(1+\kappa) \kappa^{-\frac{\kappa}{1+\kappa}}}, \quad n p \leq c \log n .
$$

If $0<c_{1}<1-\kappa$ is sufficiently small, depending only on $(\kappa, \epsilon, c)$, then there exists a universal constant $c^{\prime}>0$ so that

$$
f_{n}\left(p, \kappa, c_{1}\right) \leq c^{\prime} n^{-1-\epsilon / 2} .
$$

Proof. For $x>-1$ let $\mathcal{H}(x)$ denote the function $\mathcal{H}(x):=x-(1+x) \log (1+x)$, and note that $\mathcal{H}(x)$ is increasing for $x \leq 0$ and is decreasing otherwise. Let $0<\epsilon_{0}\left(c_{0}\right)<1,0<\epsilon_{1}\left(c_{0}\right)$ denote the unique positive solutions to

$$
\mathcal{H}\left(-\epsilon_{0}\right)=-\frac{1}{c_{0}}, \quad \mathcal{H}\left(\epsilon_{1}\right)=-\frac{2}{c_{0}} .
$$

Let $i_{0}:=\left\lceil\left(1-\epsilon_{0}\right) n p\right\rceil$ and $i_{1}:=\left\lfloor\left(1+\epsilon_{1}\right) n p\right\rfloor$, and consider first those terms in the sum (24) that satisfy either $i \leq i_{0}$ or $i \geq i_{1}$. The fact that $\left(\begin{array}{l}n \\ i\end{array}\right) \leq n^{i} / i$ ! and Stirling's formula (14) yield

$$
\left(\begin{array}{c}
n \\
i
\end{array}\right) p^{i}(1-p)^{n-i} \leq \exp (i(1+\log n p)+(n-i) \log (1-p)-\log \sqrt{2 \pi}-(i+1 / 2) \log i) .
$$

That $0<p<1$ implies $\log (1-p) \leq-p$, which in turn implies

$$
\left(\begin{array}{c}
n \\
i
\end{array}\right) p^{i}(1-p)^{n-i} \leq \exp (\Phi(i)), \quad \Phi(i):=i(1+p+\log n p)-n p-\log \sqrt{2 \pi}-i \log i .
$$

Elementary calculus demonstrates that $\Phi(i)$ increases provided $i \leq n p$. As $0<\left(1-\epsilon_{0}\right)<1$ it follows that $i_{0} \leq n p$, which together with the fact that $\left(1-\epsilon_{0}\right) n p^{2}<\log \sqrt{2 \pi}$ for $n$ sufficiently large implies

$$
\left(\begin{array}{c}
n \\
i
\end{array}\right) p^{i}(1-p)^{n-i} \leq \exp \left(\Phi\left(\left(1-\epsilon_{0}\right) n p\right)\right) \leq \exp \left(n p \mathcal{H}\left(-\epsilon_{0}\right)\right)
$$

for all $1 \leq i \leq i_{0}-1$ and all $n$ sufficiently large. The definition of $\epsilon_{0}$ and the assumption (25) then combine to imply

$$
\sum_{i=0}^{i_{0}-1}\left(\begin{array}{c}
n \\
i
\end{array}\right) p^{i}(1-p)^{n-i} \sum_{j=0}^{\left\lfloor\kappa i+c_{1} n p\right\rfloor}\left(\begin{array}{l}
n \\
j
\end{array}\right) p^{j}(1-p)^{n-j} \leq \sum_{i=0}^{i_{0}-1} n^{-(1+\epsilon)} \sum_{j=0}^{\left\lfloor\kappa i+c_{1} n p\right\rfloor}\left(\begin{array}{l}
n \\
j
\end{array}\right) p^{j}(1-p)^{n-j} \leq i_{0} n^{-(1+\epsilon)} .
$$

Next consider a term in the sum (24) that satisfies $i \geq i_{1}$. As before, the facts that $\Phi(i)$ decreases for $i \geq i_{1}$ and $\left(1+\epsilon_{1}\right) n p^{2}<\log \sqrt{2 \pi}$ if $n$ is sufficiently large imply

$$
\left(\begin{array}{c}
n \\
i
\end{array}\right) p^{i}(1-p)^{n-i} \leq \exp (\Phi(i)) \leq \exp \left(\Phi\left(\left(1+\epsilon_{1}\right) n p\right)\right) \leq \exp \left(n p \mathcal{H}\left(\epsilon_{1}\right)\right)
$$

for all $i \geq i_{1}+1$ and $n$ sufficiently large. The definition of $\epsilon_{1}$ and the assumption (25) then combine to imply

$$
\sum_{i=i_{1}+1}^{n}\left(\begin{array}{c}
n \\
i
\end{array}\right) p^{i}(1-p)^{n-i} \sum_{j=0}^{\left\lfloor\kappa i+c_{1} n p\right\rfloor}\left(\begin{array}{c}
n \\
j
\end{array}\right) p^{j}(1-p)^{n-j} \leq \sum_{i=i_{1}+1}^{n} n^{-2(1+\epsilon)} \sum_{j=0}^{\left\lfloor\kappa i+c_{1} n p\right\rfloor}\left(\begin{array}{c}
n \\
j
\end{array}\right) p^{j}(1-p)^{n-j} \leq n^{-(1+2 \epsilon)} .
$$

As $i_{0}=O(\log n)$, it follows as a consequence of these estimates that

$$
f_{n}\left(p, \kappa, c_{1}\right)=\sum_{i=i_{0}}^{i_{1}} \sum_{j=0}^{\left\lfloor\kappa i+c_{1} n p\right\rfloor}\left(\begin{array}{c}
n \\
i
\end{array}\right) p^{i}(1-p)^{n-i}\left(\begin{array}{c}
n \\
j
\end{array}\right) p^{j}(1-p)^{n-j}+O\left(\frac{\log n}{n^{1+\epsilon}}\right) .
$$

Now let $G(i, j)$ denote the function,

$$
\begin{aligned}
G(i, j) & :=\left(\begin{array}{c}
n \\
i
\end{array}\right) p^{i}(1-p)^{n-i}\left(\begin{array}{c}
n \\
j
\end{array}\right) p^{j}(1-p)^{n-j}=\mathrm{e}^{\Phi_{0}(i)} \mathrm{e}^{\Phi_{0}(j)} \\
\Phi_{0}(i) & :=\log \Gamma(n+1)+i \log \frac{p}{1-p}+n \log (1-p)-(\log \Gamma(i+1)+\log \Gamma(n-i+1))
\end{aligned}
$$


and let $S$ denote the constraint set

$$
S:=\left\{(i, j) \in \mathbb{R}_{+}^{2}:\left(1-\epsilon_{0}\right) n p \leq i \leq\left(1+\epsilon_{1}\right) n p, 0 \leq j \leq \kappa i+c_{1} n p\right\} .
$$

For all indices $i, j$ such that $(i, j) \in S$ it trivially holds that

$$
G(i, j) \leq G_{*}:=\max _{(i, j) \in S} G(i, j)
$$

As $n p=O(\log n), i_{0}=O(\log n)$ and $i_{1}=O(\log n)$ it follows that

$$
\sum_{i=i_{0}}^{i_{1}} \sum_{j=0}^{\left\lfloor\kappa i+c_{1} n p\right\rfloor}\left(\begin{array}{l}
n \\
i
\end{array}\right) p^{i}(1-p)^{n-i}\left(\begin{array}{c}
n \\
j
\end{array}\right) p^{j}(1-p)^{n-j} \leq O\left(\log ^{2} n\right) G_{*} .
$$

Suppose that the maximum $G_{*}$ occurs on the boundary $\partial S$ of the constraint set. This leaves four cases to consider. In the first two cases, if $i=\left(1-\epsilon_{0}\right) n p$ or $i=\left(1+\epsilon_{1}\right) n p$ the preceeding arguments imply that

$$
\begin{aligned}
& G\left(\left(1-\epsilon_{0}\right) n p, j\right) \leq \exp \left\{\Phi\left(n p \mathcal{H}\left(-\epsilon_{0}\right)\right)\right\} \leq n^{-(1+\epsilon)} \\
& G\left(\left(1+\epsilon_{1}\right) n p, j\right) \leq \exp \left\{\Phi\left(n p \mathcal{H}\left(\epsilon_{1}\right)\right)\right\} \leq n^{-2(1+\epsilon)}
\end{aligned}
$$

In the third case, i.e. $j=0$ and $\left(1-\epsilon_{0}\right) n p \leq i \leq\left(1+\epsilon_{1}\right) n p$, the maximum satisfies

$$
G(i, 0) \leq(1-p)^{n}=\mathrm{e}^{\log (1-p) n} \leq \mathrm{e}^{-n p} \leq n^{-(1+\epsilon) c_{0}(\kappa)}
$$

which decays faster than $n^{-(1+\epsilon)}$ due to the fact that $c_{0}(\kappa)>1$ for $0<\kappa<1$ by definition.

The final case proves the most difficult. In this remaining case it holds that $j=\kappa i+c_{1} n p$ and that

$$
\begin{aligned}
& G\left(i, \kappa i+c_{1} n p\right)=\mathrm{e}^{\Phi_{1}(i)}, \quad \Phi_{1}(i):=2 \log \Gamma(n+1)+\left[i(1+\kappa)+c_{1} n p\right] \log \frac{p}{1-p}+ \\
& 2 n \log (1-p)-\left[\log \Gamma(i+1)+\log \Gamma\left(\kappa i+c_{1} n p+1\right)+\log \Gamma(n-i+1)+\log \Gamma\left(n-\kappa i-c_{1} n p+1\right)\right] .
\end{aligned}
$$

If a maximum of $\Phi_{1}(i)$ occurs between $i_{0}$ and $i_{1}$ then the maximum must occur when

$$
(1+\kappa) \log \frac{p}{1-p}=\Psi^{0}(i+1)+\kappa \Psi^{0}\left(\kappa i+c_{1} n p+1\right)-\kappa \Psi^{0}\left(n-\kappa i-c_{1} n p+1\right)-\Psi^{0}(n-i+1):=\chi(i) .
$$

Indeed, as $\Psi^{0}(z)>0$ increases for $z>0$, if a solution $i_{0}<i_{*}<i_{1}$ to (27) exists then it is unique and is a maximum of $\Phi_{1}(i)$. Let $\delta\left(\kappa, c_{1}\right)$ denote the unique, positive solution to $\delta\left(\delta \kappa+c_{1}\right)^{\kappa}=1$ and set $i_{u}=2 \delta\left(\kappa, c_{1}\right) n p$. From the digamma estimate (18) it follows that

$$
\begin{aligned}
\chi\left(i_{u}\right) & \geq \log \left(\frac{i_{u}\left(\kappa i_{u}+c_{1} n p\right)^{\kappa}}{\left(n-\kappa i_{u}-c_{1} n p+1\right)^{\kappa}\left(n-i_{u}+1\right)}\right) \\
& =\log \left(p^{(1+\kappa)} \frac{2 \delta\left(\kappa, c_{1}\right)\left(\kappa 2 \delta\left(\kappa, c_{1}\right)+c_{1}\right)^{\kappa}}{\left(1-\kappa 2 \delta\left(\kappa, c_{1}\right) p-c_{1} p+\frac{1}{n}\right)^{\kappa}\left(1-2 \delta\left(\kappa, c_{1}\right) p+\frac{1}{n}\right)}\right)>(1+\kappa) \log \frac{p}{1-p}
\end{aligned}
$$

for all $n$ sufficiently large. The last inequality follows due to the fact that $2 \delta\left(\kappa, c_{1}\right)\left(\kappa 2 \delta\left(\kappa, c_{1}\right)+c_{1}\right)^{\kappa}>1$ is constant in $n$, so when $p=o(1)$ the coefficient of $p^{1+\kappa}$ on the left hand side (which asymptotically equals $\left.2 \delta\left(\kappa, c_{1}\right)\left(\kappa 2 \delta\left(\kappa, c_{1}\right)+c_{1}\right)^{\kappa}\right)$ always exceeds the coefficient of $p^{1+\kappa}$ (which equals one) on the right hand side. As the right hand side, i.e. $\chi(i)$, of $(27)$ is increasing, it follows that $i_{*}<i_{u}$ for all $n$ sufficiently large. Defining $i_{l}:=\delta\left(\kappa, c_{1}\right) n p / 2$, it follows in a similar fashion that $i_{l}<i_{*}$ for all $n$ sufficiently large as well. In particular, $i_{*}=\Theta(n p)$. Write $i_{*}=\delta(n) n p$, and note that the critical point equation $(27)$ and the digamma estimate (18) also imply

$$
\log \left(\frac{\left(i_{*}+1\right)\left(\kappa i_{*}+c_{1} n p+1\right)^{\kappa}}{\left(n-\kappa i_{*}-c_{1} n p\right)^{\kappa}\left(n-i_{*}\right)}\right) \geq(1+\kappa) \log \frac{p}{1-p} \geq \log \left(\frac{i_{*}\left(\kappa i_{*}+c_{1} n p\right)^{\kappa}}{\left(n-\kappa i_{*}-c_{1} n p+1\right)^{\kappa}\left(n-i_{*}+1\right)}\right) .
$$

These inequalities imply that

$$
\frac{\left(\delta(n)+\frac{1}{n p}\right)\left(\kappa \delta(n)+c_{1}+\frac{1}{n p}\right)^{\kappa}}{\left(1-\kappa \delta(n) p-c_{1} p\right)^{\kappa}(1-\delta(n) p)} \geq \frac{1}{(1-p)^{\kappa+1}} \geq \frac{\delta(n)\left(\kappa \delta(n)+c_{1}\right)^{\kappa}}{\left(1-\kappa \delta(n) p-c_{1} p+\frac{1}{n}\right)^{\kappa}\left(1-\delta(n) p+\frac{1}{n}\right)} .
$$


From the fact that $p=o(1)$, the fact that $n p \rightarrow \infty$ and the fact that $\delta(n)$ is a bounded sequence, by passing to the limit in the previous expression it follows that any limit point $\delta_{\infty}$ of the sequence $\delta(n)$ must satisfy

$$
\delta_{\infty}\left(\kappa \delta_{\infty}+c_{1}\right)^{\kappa} \geq 1 \geq \delta_{\infty}\left(\kappa \delta_{\infty}+c_{1}\right)^{\kappa} .
$$

In particular, $\delta_{\infty}$ is the unique solution to $\delta\left(\kappa \delta+c_{1}\right)^{\kappa}=1$. As any convergent subsequence of $\delta(n)$ must converge to $\delta\left(\kappa, c_{1}\right)$, i.e. the unique solution to $\delta\left(\kappa \delta+c_{1}\right)^{\kappa}=1$, it follows that in fact $\delta(n)=\delta\left(\kappa, c_{1}\right)+o(1)$.

Write the value at such a maximum as $\mathrm{e}^{\Phi_{1}\left(i_{*}\right)}$ with $\Phi_{1}\left(i_{*}\right)$ defined above. Using Stirling's approximation (15) it follows that $\Phi_{1}\left(i_{*}\right)=$

$$
\begin{aligned}
& n \log \left(\frac{n^{2}}{\left(n-i_{*}\right)\left(n-\kappa i_{*}-c_{1} n p\right)}\right)+\left[(1+\kappa) i_{*}+c_{1} n p\right] \log \frac{p}{1-p}+2 n \log (1-p)-i_{*} \log \left(\frac{i_{*}}{n-i_{*}}\right) \\
& \frac{1}{2} \log \left(\frac{n^{2}}{i_{*}\left(\kappa i_{*}+c_{1} n p\right)\left(n-i_{*}\right)\left(n-\kappa i_{*}-c_{1} n p\right)}\right)-\left(\kappa i_{*}+c_{1} n p\right) \log \left(\frac{\kappa i_{*}+c_{1} n p}{n-\kappa i_{*}-c_{1} n p}\right)+O(1) .
\end{aligned}
$$

Now recall that $i_{*}=\delta(n) n p$ for $\delta(n)=\delta\left(\kappa, c_{1}\right)+o(1)$, so that

$$
\begin{gathered}
\frac{1}{2} \log \left(\frac{n^{2}}{i_{*}\left(\kappa i_{*}+c_{1} n p\right)\left(n-i_{*}\right)\left(n-\kappa i_{*}-c_{1} n p\right)}\right)=-\log n p+O(1) \\
n \log \left(\frac{n^{2}}{\left(n-i_{*}\right)\left(n-\kappa i_{*}-c_{1} n p\right)}\right)=-n\left(\log (1-\delta(n) p)+\log \left(1-\kappa \delta(n) p-c_{1} p\right)\right)
\end{gathered}
$$

That $\log (1-p)=-p+O\left(p^{2}\right)$ and $n p=O(\log n)$ imply $2 n \log (1-p)=-2 n p+o(1)$, which yields $\Phi_{1}\left(i_{*}\right)=$ $O(1)-\log n p+$

$$
\left[\delta(n)(1+\kappa)+c_{1}-2\right] n p+\left[(1+\kappa) i_{*}+c_{1} n p\right] \log \frac{p}{1-p}-i_{*} \log \left(\frac{i_{*}}{n-i_{*}}\right)-\left(\kappa i_{*}+c_{1} n p\right) \log \left(\frac{\kappa i_{*}+c_{1} n p}{n-\kappa i_{*}-c_{1} n p}\right) .
$$

Appealing to the asymptotic formula (17) for the digamma function then shows

$$
\begin{aligned}
& \log \left(i_{*}\right)-\log \left(n-i_{*}\right)=\Psi^{0}\left(i_{*}+1\right)-\Psi^{0}\left(n-i_{*}+1\right)+O\left(\frac{1}{i_{*}}\right)+O\left(\frac{1}{n-i_{*}}\right) \\
& i_{*}\left\{\log \left(i_{*}\right)-\log \left(n-i_{*}\right)\right\}=i_{*}\left\{\Psi^{0}\left(i_{*}+1\right)-\Psi^{0}\left(n-i_{*}+1\right)\right\}+O(1) \\
& \kappa i_{*}\left\{\log \left(\kappa i_{*}+c_{1} n p\right)-\log \left(n-\kappa i_{*}-c_{1} n p\right)\right\}=i_{*}\left\{\kappa \Psi^{0}\left(\kappa i_{*}+c_{1} n p+1\right)-\kappa \Psi^{0}\left(n-\kappa i_{*}-c_{1} n p+1\right)\right\}+O(1) .
\end{aligned}
$$

As a consequence, the critical point equation (27) implies

$$
\begin{aligned}
& i_{*} \log \left(\frac{i_{*}}{n-i_{*}}\right)+\kappa i_{*} \log \left(\frac{\kappa i_{*}+c_{1} n p}{n-\kappa i_{*}-c_{1} n p}\right)=i_{*}(1+\kappa) \log \frac{p}{1-p}+O(1), \\
& \Phi_{1}\left(i_{*}\right)=\left[(1+\kappa) \delta(n)+c_{1}-2\right] n p-\log n p+c_{1} n p \log \left(\frac{1-\kappa \delta(n) p-c_{1} p}{(1-p)\left(\delta(n) \kappa+c_{1}\right)}\right)+O(1) .
\end{aligned}
$$

That $f(\delta):=\delta\left(\kappa \delta+c_{1}\right)^{\kappa}$ increases with $\delta$ and $f\left(\kappa^{-\frac{\kappa}{1+\kappa}}\right)>1$ implies $\delta\left(\kappa, c_{1}\right)<\kappa^{-\frac{\kappa}{1+\kappa}}$. The fact that $\delta(n)=\delta\left(\kappa, c_{1}\right)+o(1)$ and the hypothesis $(25)$ then combine to demonstrate

$$
\Phi_{1}\left(i_{*}\right) \leq\left(-\frac{1}{c_{0}(\kappa)}+c_{1}\left(1-\log \left(\kappa \delta\left(\kappa, c_{1}\right)+c_{1}\right)\right)+o(1)\right) n p \leq-(1+2 \epsilon / 3) \log n
$$

provided $c_{1}$ is sufficiently small (depending on $(\epsilon, \kappa, c)$ ). Thus, if a maximum $i_{*}$ occurs between $i_{0}$ and $i_{1}$ then it must satisfy

$$
G\left(i_{*}, \kappa i_{*}+c_{1} n p\right) \leq c^{\prime} n^{-(1+\epsilon / 2)} .
$$

As a consequence, in all four cases there exists a $c^{\prime}>0$ so that the maximum $G_{*}$ satisfies $G^{*} \leq c^{\prime} n^{-(1+2 \epsilon / 3)}$.

In summary, provided

$$
G_{*}:=\max _{(i, j) \in S} G(i, j)=\max _{(i, j) \in \partial S} G(i, j)
$$


the estimate

$$
\begin{aligned}
f_{n}\left(p, \kappa, c_{1}\right) & =\sum_{i=i_{0}}^{i_{1}} \sum_{j=0}^{\left\lfloor\kappa i+c_{1} n p\right\rfloor}\left(\begin{array}{c}
n \\
i
\end{array}\right) p^{i}(1-p)^{n-i}\left(\begin{array}{c}
n \\
j
\end{array}\right) p^{j}(1-p)^{n-j}+O\left(\frac{\log n}{n^{1+\epsilon}}\right) \\
& \leq O\left(\log ^{2} n\right) G_{*}+O\left(\frac{\log n}{n^{1+\epsilon}}\right) \leq O\left(\log ^{2} n\right) n^{-(1+2 \epsilon / 3)}
\end{aligned}
$$

holds for all $n$ sufficiently large. It therefore suffices to establish (28), i.e. that the maximum of $G(i, j)$ occurs along the boundary $\partial S$ of the constraint set $S:=\left\{(i, j):\left(1-\epsilon_{0}\right) n p \leq i \leq\left(1+\epsilon_{1}\right) n p, 0 \leq j \leq \kappa i+c_{1} n p\right\}$. If the maximum $G_{*}$ were attained in the interior of the at some point $\left(i_{*}, j_{*}\right)$ then both $\Phi_{0}^{\prime}\left(i_{*}\right)=\Phi_{0}^{\prime}\left(j_{*}\right)=0$ would simultaneously hold. Differentiating $\Phi_{0}$ shows that this would imply

$$
\log \frac{p}{1-p}=\Psi^{0}\left(i_{*}+1\right)-\Psi^{0}\left(n-i_{*}+1\right), \quad \log \frac{p}{1-p}=\Psi^{0}\left(j_{*}+1\right)-\Psi^{0}\left(n-j_{*}+1\right) .
$$

As $\Psi^{0}(i+1)-\Psi^{0}(n-i+1)$ is strictly increasing, this would imply $i_{*}=j_{*}$ as a consequence. Moreover, the digamma estimate (18) implies $n p-1 \leq i_{*}=j_{*} \leq p(n+1)$, which since $\kappa+c_{1}<1$ yields in turn

$$
\kappa i_{*}+c_{1} n p \leq \kappa(n+1) p+c_{1} n p=\left(\kappa+c_{1}\right) n p+p<n p-1 \leq j_{*}
$$

for all $n$ sufficiently large. In other words, $\left(i_{*}, j_{*}\right) \notin S$ and the maximum must occur on $\partial S$.

\subsection{Proof of the Main Result}

We now have all the ingredients necessary to establish the threshold for stability of the compromise equilibrium. If $n p \geq(1+\epsilon) c_{0}(\kappa) \log n$ then lemma 3.3 suffices to guarantee that each diagonal entry $d_{i i}$ of the diagonal component (20) satisfies

$$
d_{i i} \leq-c_{1} N p
$$

with probability at least $1-c^{\prime} n^{-(1+\epsilon / 2)}$. As a consequence, the union bound implies that there exists $c_{1}>0$ so that

$$
\lambda_{1}(D) \leq-c_{1} N p
$$

asymptotically almost surely. The reduction furnished by lemma 3.1 then implies stability asymptotically almost surely.

The converse direction proves slightly more difficult due to the fact that the diagonal entries $d_{i i}$ exhibit a mild dependence. This dependence results from the undirected graph. Nevertheless, a standard technique easily adapts to the present situation and allows us to handle this lack of independence. If $n p \leq(1-\epsilon) \log n$ then a previous result [49] already implies instability asymptotically almost surely. We therefore may as well assume that $(1-\epsilon) \log n \leq n p \leq c_{0}(\kappa)(1-\epsilon) \log n$, so that lemma 3.2 applies and there exists a $c_{1}>0$ sufficiently small so that

$$
d_{i i} \geq c_{1} n p
$$

with probability at least $c^{\prime} n^{-1+\epsilon / 2}$ for any given diagonal entry. Let $X_{i}:=\mathbf{1}_{\left\{d_{i i} \geq c_{1} n p\right\}}$ denote the indicator of such an event and define

$$
N_{0}:=\sum_{i=1}^{n} X_{i}
$$

as the total number of such events that occur over the first $n$ diagonal entries. Let $\mu_{0}:=\mathbb{E}\left(N_{0}\right)=n f_{n}\left(p, \kappa, c_{1}\right)$ denote the expected number of such entries. Chebyshev's inequality then implies that (writing $f_{n}$ as shorthand for $\left.f_{n}\left(p, \kappa, c_{1}\right)\right)$ for any $\gamma>0$ the inequality

$$
\mathbb{P}\left(\left|N_{0}-\mu_{0}\right|>\gamma n f_{n}\right) \leq \frac{\operatorname{Var}\left(N_{0}\right)}{\gamma^{2} n^{2} f_{n}^{2}}
$$

holds. The variance satisfies

$$
\operatorname{Var}\left(N_{0}\right)=\sum_{i=1}^{n} \operatorname{Var}\left(X_{i}\right)+2 \sum_{i=1}^{n} \sum_{j>i} \operatorname{Cov}\left(X_{i}, X_{j}\right)=n f_{n}\left(1-f_{n}\right)+2 \sum_{i=1}^{n} \sum_{j>i} \operatorname{Cov}\left(X_{i}, X_{j}\right),
$$


whereas the covariance satisfies

$$
\operatorname{Cov}\left(X_{i}, X_{j}\right)=\mathbb{P}\left(X_{i}=1 \cap X_{j}=1\right)-f_{n}^{2} .
$$

Recalling the definition of $D$ in (20) shows that we may decompose

$$
d_{i i}=\kappa \sum_{k=1}^{n} a_{i k}-\sum_{k=1}^{n} b_{i k} \quad d_{j j}=\kappa \sum_{k=1}^{n} a_{j k}-\sum_{k=1}^{n} b_{j k},
$$

which obviates the fact that the only dependence between $d_{i i}$ and $d_{j j}$ occurs via the entry $a_{i j}$; indeed, the entries $\left\{a_{i k}\right\}_{k \neq j}^{n},\left\{a_{j k}\right\}_{k \neq i}^{n}$ and $\left\{b_{i j}\right\}_{i j=1}^{n}$ are independent. With this in mind, define

$$
\tilde{d}_{i i}:=\kappa \sum_{k \neq j}^{n} a_{i k}-\sum_{k=1}^{n} b_{i k}=d_{i i}-\kappa a_{i j}
$$

and define $\tilde{d}_{j j}$ similarly. Conditioning on the possible values of $a_{i j} \in\{0,1\}$ shows

$$
\mathbb{P}\left(X_{i}=1 \cap X_{j}=1\right)=\mathbb{P}\left(\tilde{d}_{i i} \geq c_{1} n p-\kappa\right) \mathbb{P}\left(\tilde{d}_{j j} \geq c_{1} n p-\kappa\right) p+\mathbb{P}\left(\tilde{d}_{i i} \geq c_{1} n p\right) \mathbb{P}\left(\tilde{d}_{j j} \geq c_{1} n p\right)(1-p) .
$$

Note that we may write

$$
\mathbb{P}\left(\tilde{d}_{i i} \geq c_{1} n p-\kappa\right)=\sum_{i=1}^{n} \sum_{j=0}^{\left\lfloor\kappa i-c_{1} n p\right\rfloor}\left(\begin{array}{c}
n-1 \\
i-1
\end{array}\right)\left(\begin{array}{c}
n \\
j
\end{array}\right) p^{i-1}(1-p)^{n-i} p^{j}(1-p)^{n-j} .
$$

As in the proof of lemma 3.3 , there exists an $i_{1}=O(n p)$ sufficiently large so that

$$
\begin{aligned}
& \sum_{i=1}^{n} \sum_{j=0}^{\left\lfloor\kappa i-c_{1} n p\right\rfloor}\left(\begin{array}{c}
n-1 \\
i-1
\end{array}\right)\left(\begin{array}{c}
n \\
j
\end{array}\right) p^{i-1}(1-p)^{n-i} p^{j}(1-p)^{n-j}=\sum_{i=1}^{i_{1}} \sum_{j=0}^{\left\lfloor\kappa i-c_{1} n p\right\rfloor}+O\left(\frac{1}{n}\right) \\
& f_{n}=\sum_{i=1}^{i_{1}} \sum_{j=0}^{\left\lfloor\kappa i-c_{1} n p\right\rfloor}\left(\begin{array}{c}
n \\
i
\end{array}\right)\left(\begin{array}{c}
n \\
j
\end{array}\right) p^{i}(1-p)^{n-i} p^{j}(1-p)^{n-j}+O\left(\frac{1}{n}\right) .
\end{aligned}
$$

For $i=O(n p)$ it holds that

$$
\left(\begin{array}{c}
n-1 \\
i-1
\end{array}\right) p^{i-1}(1-p)^{n-i}=O(1)\left(\begin{array}{c}
n \\
i
\end{array}\right) p^{i}(1-p)^{n-i}
$$

which implies

$$
\mathbb{P}\left(\tilde{d}_{i i} \geq c_{1} n p-\kappa\right) \leq O(1) f_{n}+O\left(\frac{1}{n}\right) .
$$

The fact that $\left\{\tilde{d}_{i i} \geq c_{1} n p\right\} \subset\left\{X_{i}=1\right\}$ implies

$$
\mathbb{P}\left(\tilde{d}_{i i} \geq c_{1} n p\right) \leq f_{n},
$$

which yields as a consequence the estimate

$$
\mathbb{P}\left(X_{i}=1 \cap X_{j}=1\right) \leq f_{n}^{2}+O(1) f_{n}^{2} p+O\left(\frac{p}{n}\right)=f_{n}^{2}+O(1) f_{n}^{2} p .
$$

The last line follows as a consequence of lemma 3.2. Substituting this estimate into the covariance, we conclude that

$$
\operatorname{Var}\left(N_{0}\right) \leq n f_{n}+O(1) n^{2} f_{n}^{2} p
$$

This estimate combines with (29), the fact that $n p=O(\log n)$ and the fact that $f_{n} \geq c^{\prime} n^{-1+\epsilon / 2}$ to show that for any fixed $\gamma>0$ the inequality

$$
\mathbb{P}\left(\left|N_{0}-\mu_{0}\right|>\gamma n f_{n}\right) \leq \frac{1+O(1) \log n}{\gamma^{2} n f_{n}} \lesssim \frac{\log n}{n^{\epsilon / 2}}
$$

holds. In particular, if $\gamma=1 / 2$ this yields $N_{0} \geq n f_{n} / 2$ asymptotically almost surely. Thus the stability matrix has at least $O\left(n^{\epsilon / 2}\right)$ positive diagonal entries, and so the compromise solution is unstable asymptotically almost surely. 


\section{Problem Statement in Higher Dimensions}

We begin this section by describing the stability matrix and the associated linear stability condition, i.e the analogue of (19), that arises by linearizing (2) around the two-dimensional simplex. We may then state the general $d$-dimensional problem as a straightforward generalization of the two-dimensional case.

The two-dimensional version of the compromise model consists of three equal-sized groups of individuals that occupy the vertices of a regular, two-dimensional simplex. Specifically, let $\mathbf{p}_{1}=(1,0)^{t}, \mathbf{p}_{2}=$ $(-1 / 2, \sqrt{3} / 2)^{t}$ and $\mathbf{p}_{3}=(-1 / 2,-\sqrt{3} / 2)^{t}$ denote the vertices of an equilateral triangle. Let $n$ denotes the number of individuals in each group and let $\mathbf{v}_{i} \in \mathbb{R}^{2}$ denote the position of the $i^{\text {th }}$ individual. Without loss of generality, we may order the individuals in such a fashion so that

$$
\mathbf{v}_{1}=\cdots=\mathbf{v}_{n}=\mathbf{p}_{1}, \quad \mathbf{v}_{n+1}=\cdots=\mathbf{v}_{2 n}=\mathbf{p}_{2}, \quad \mathbf{v}_{2 n+1}=\cdots=\mathbf{v}_{N}=\mathbf{p}_{3} \quad N=3 n .
$$

Let $G_{1}=\{1, \ldots, n\}, G_{2}=\{n+1, \ldots, 2 n\}$ and $G_{3}=\{2 n+1, \ldots, N\}$ denote the corresponding partition of the vertices into the three groups. Finally, let

$$
\mathbf{x}=\left(\mathbf{p}_{2}-\mathbf{p}_{1}\right) /\left|\mathbf{p}_{2}-\mathbf{p}_{1}\right|, \quad \mathbf{y}=\left(\mathbf{p}_{3}-\mathbf{p}_{1}\right) /\left|\mathbf{p}_{3}-\mathbf{p}_{1}\right|, \quad \mathbf{z}=\left(\mathbf{p}_{2}-\mathbf{p}_{3}\right) /\left|\mathbf{p}_{2}-\mathbf{p}_{3}\right| .
$$

From an undirected Erdős-Rényi random graph $\mathcal{G}(N, p)$ with edges $\left\{e_{i j}\right\}$ and adjacency matrix $E$, we first form four $2 N \times 2 N$ generalized adjacency matrices $A(\mathrm{Id}), A\left(\mathbf{x x}^{t}\right), A\left(\mathbf{y} \mathbf{y}^{t}\right)$ and $A\left(\mathbf{z z}^{t}\right)$ in such a way so that

$$
A\left(\mathbf{x x}^{t}\right)=E \otimes \mathbf{x x}^{t} \quad A\left(\mathbf{y} \mathbf{y}^{t}\right)=E \otimes \mathbf{y y}^{t} \quad A\left(\mathbf{z z}^{t}\right)=E \otimes \mathbf{z z}^{t} \quad A(\mathrm{Id})=E \otimes \mathrm{Id}
$$

where $A \otimes B$ denotes the Kronecker product of two matrices. In more explicit terms, given a $2 \times 2$ symmetric matrix $M$, we partition $A(M)$ into $2 \times 2$ blocks $A_{i j}(M)$ :

$$
A(M)=\left(\begin{array}{cccc}
A_{11}(M) & A_{12}(M) & \cdots & A_{1 N}(M) \\
A_{21}(M) & A_{22}(M) & \cdots & A_{2 N}(M) \\
\vdots & \vdots & \ddots & \vdots \\
A_{N 1}(M) & A_{N 2}(M) & \cdots & A_{N N}(M)
\end{array}\right)
$$

For $j \geq i$ we set $A_{i j}(M)=M$ if $e_{i j}=1$ and $A_{i j}=0$ otherwise. For $j<i$ we set $A_{i j}(M)=A_{j i}^{t}(M)$, or in other words we define the lower triangle via symmetry. The matrices $A(\mathrm{Id}), A\left(\mathbf{x x}^{t}\right), A\left(\mathbf{y y}^{t}\right)$ and $A\left(\mathbf{z z}^{t}\right)$ constructed in this manner agree with the generalized adjacency matrices (31) defined via the sub-blocks Id, $\mathbf{x} \mathbf{x}^{t}, \mathbf{y} \mathbf{y}^{t}$ and $\mathbf{z z} \mathbf{z}^{t}$, respectively.

Next, we decompose each generalized adjacency matrix $A(M)$ into $2 n \times 2 n$ blocks $A^{k l}(M)$ that correspond to the interactions between group $G_{k}$ and group $G_{l}$ :

$$
A(M)=\left(\begin{array}{lll}
A^{11}(M) & A^{12}(M) & A^{13}(M) \\
A^{21}(M) & A^{22}(M) & A^{23}(M) \\
A^{31}(M) & A^{32}(M) & A^{33}(M)
\end{array}\right) .
$$

Note that $A^{k l}(M)=\left(A^{l k}(M)\right)^{t}$ due symmetry. While only a portion of each generalized adjacency matrix appears in the linear stability matrix, referencing the full generalized adjacency matrices will prove useful in deriving estimates. We therefore denote the relevant portions of each matrix as follows -

$$
\begin{array}{rlrl}
B(\mathrm{Id}) & =\left(\begin{array}{ccc}
A^{11}(\mathrm{Id}) & 0 & 0 \\
0 & A^{22}(\mathrm{Id}) & 0 \\
0 & 0 & A^{33}(\mathrm{Id})
\end{array}\right) & B\left(\mathbf{x x ^ { t }}\right)=\left(\begin{array}{ccc}
0 & A^{12}\left(\mathbf{x} \mathbf{x}^{t}\right) & 0 \\
A^{21}\left(\mathbf{x} \mathbf{x}^{t}\right) & 0 & 0 \\
0 & 0 & 0
\end{array}\right) \\
B\left(\mathbf{y} \mathbf{y}^{t}\right)=\left(\begin{array}{ccc}
0 & 0 & A^{13}\left(\mathbf{y} \mathbf{y}^{t}\right) \\
0 & 0 & 0 \\
A^{31}\left(\mathbf{y} \mathbf{y}^{t}\right) & 0 & 0
\end{array}\right) & B\left(\mathbf{z z}^{t}\right)=\left(\begin{array}{ccc}
0 & 0 & 0 \\
0 & 0 & A^{23}\left(\mathbf{z z}^{t}\right) \\
0 & A^{32}\left(\mathbf{z z}^{t}\right) & 0
\end{array}\right) .
\end{array}
$$

Lastly, using each $B(M)$ we define corresponding generalized Laplacian matrices in the straightforward way, i.e. by using block-diagonal row sums. In other words, we may define these matrices by noting that, analogously to $A(M)$, each $B(M)$ decomposes into a $2 \times 2$ block-matrix structure according to (32). We can 
therefore define a corresponding block-diagonal matrix $D(M)$ with $2 \times 2$ blocks along the diagonal by using row sums of the $2 \times 2$ blocks in $B(M)$,

$$
D_{i i}(M)=\sum_{j=1}^{N} B_{i j}(M) \quad D_{i j}(M)=0 \text { if } i \neq j .
$$

We then define the Laplacian matrix $L(M)=D(M)-B(M)$ for each $M \in\left\{\operatorname{Id}, \mathbf{x} \mathbf{x}^{t}, \mathbf{y} \mathbf{y}^{t}, \mathbf{z z}^{t}\right\}$, and for $\kappa>0$ consider the random stability matrix

$$
L:=\kappa L(\mathrm{Id})-\left(L\left(\mathbf{x} \mathbf{x}^{t}\right)+L\left(\mathbf{y} \mathbf{y}^{t}\right)+L\left(\mathbf{z} \mathbf{z}^{t}\right)\right) .
$$

Linearizing (2) around the two-dimensional simplex equilibrium produces a random matrix of precisely this form.

Like the one-dimensional case, this stability matrix necessarily has a non-trivial nullspace due to the underlying translation and rotation invariances inherent in the ODE system. Analogously to (19), we must therefore account for these zero eigenvalues when definining our notion of stability. For any $\mathbf{w} \in \mathbb{R}^{2}$ put $\mathbf{v}_{c}=(\mathbf{w}, \mathbf{w}, \ldots, \mathbf{w})^{t} \in \mathbb{R}^{2 N}$, i.e. a "constant vector." A straightforward computation reveals that $L \mathbf{v}_{c}=0$. Additionally, set

$$
\mathbf{v}_{r}=\left(\mathbf{p}_{1}^{\perp}, \ldots, \mathbf{p}_{1}^{\perp}, \mathbf{p}_{2}^{\perp}, \ldots, \mathbf{p}_{2}^{\perp}, \mathbf{p}_{3}^{\perp}, \ldots, \mathbf{p}_{3}^{\perp}\right)^{t} \in \mathbb{R}^{2 N}
$$

where each $\mathbf{p}_{i}^{\perp}$ appears exactly $n$ times, and for a given $\mathbf{w}=\left(w_{1}, w_{2}\right)^{t} \in \mathbb{R}^{2}$ we define $\mathbf{w}^{\perp}=\left(-w_{2}, w_{1}\right)^{t}$. Using the antisymmetry $\left\langle\mathbf{p}_{i}^{\perp}, \mathbf{p}_{j}\right\rangle=-\left\langle\mathbf{p}_{j}^{\perp}, \mathbf{p}_{i}\right\rangle$ and the definitions of $\mathbf{x}, \mathbf{y}, \mathbf{z}$ then shows $L \mathbf{v}_{r}=0$ as well. If $V$ denotes the subspace spanned by $\mathbf{v}_{c}$ and $\mathbf{v}_{r}$, we therefore wish to know when the stability condition

$$
\max _{\mathbf{v} \in V^{\perp},\|\mathbf{v}\|=1}\langle\mathbf{v}, L \mathbf{v}\rangle<0
$$

holds asymptotically almost surely.

Remark 4.1. The construction of the stability matrix for the d-dimensional simplex solutions follows analogously. We let $\mathbf{p}_{1}, \ldots, \mathbf{p}_{d+1} \in \mathbb{R}^{d}$ denote the vertices of a regular simplex and form $k_{d}:=d(d+1) / 2$ vectors $\mathbf{x}_{k}, k=1 \cdots k_{d}$ from all possible differences between unique pairs of vertices. We then form the corresponding $n d \times n d$ generalized adjacency matrices $E \otimes \mathrm{Id}$ and $E \otimes \mathbf{x}_{k} \mathbf{x}_{k}^{t}$ and construct the Laplacian matrices $L(\mathrm{Id})$ and $L\left(\mathbf{x}_{k} \mathbf{x}_{k}^{t}\right)$ in a similar fashion to the two dimensional case. The relevant random matrix $L=\kappa L(\mathrm{Id})-\sum_{k=1}^{k_{d}} L\left(\mathbf{x}_{k} \mathbf{x}_{k}^{t}\right)$ necessarily has a $k_{d}$ dimensional nullspace. The first $d$ result from the constant vectors $\mathbf{v}_{c}=(\mathbf{w}, \mathbf{w}, \ldots, \mathbf{w})^{t} \in \mathbb{R}^{N d(d+1)}$ and the remaining $d(d-1) / 2$ vectors $\mathbf{v}_{r}$ result from the total possible independent rotations of the simplex. The stability analysis then proceeds by estimating the equivalent of $(36)$.

\subsection{Reduction to the Diagonal Component}

We now turn to the task of establishing the existence of a critical scaling $N p=O(\log N)$ for stability in the two-dimensional compromise model. As in the one-dimensional case, we first reduce the task to understanding the (block) diagonal of the corresponding stability matrix. Corresponding to the block decomposition (34), we may decompose each of the diagonal matrices $D(\mathrm{Id}), D\left(\mathbf{x} \mathbf{x}^{t}\right), D\left(\mathbf{y} \mathbf{y}^{t}\right)$ and $D\left(\mathbf{z z} \mathbf{z}^{t}\right)$ as

$$
\begin{array}{rlrl}
D(\mathrm{Id}) & =\left(\begin{array}{ccc}
D^{11}(\mathrm{Id}) & 0 & 0 \\
0 & D^{22}(\mathrm{Id}) & 0 \\
0 & 0 & D^{33}(\mathrm{Id})
\end{array}\right) & D\left(\mathbf{x} \mathbf{x}^{t}\right)=\left(\begin{array}{ccc}
D^{12}\left(\mathbf{x} \mathbf{x}^{t}\right) & 0 & 0 \\
0 & D^{21}\left(\mathbf{x} \mathbf{x}^{t}\right) & 0 \\
0 & 0 & 0
\end{array}\right) \\
D\left(\mathbf{y y}^{t}\right)=\left(\begin{array}{ccc}
D^{13}\left(\mathbf{y} \mathbf{y}^{t}\right) & 0 & 0 \\
0 & 0 & 0 \\
0 & 0 & D^{31}\left(\mathbf{y} \mathbf{y}^{t}\right)
\end{array}\right) & D\left(\mathbf{z z}^{t}\right)=\left(\begin{array}{ccc}
0 & 0 & 0 \\
0 & D^{23}\left(\mathbf{z z}^{t}\right) & 0 \\
0 & 0 & D^{32}\left(\mathbf{z z}^{t}\right)
\end{array}\right) .
\end{array}
$$

Recall that we use $\mathbf{e}_{1}:=(1,0,1,0, \ldots, 1,0)^{t} \in \mathbb{R}^{2 n}$ and $\mathbf{e}_{2}=(0,1,0,1, \ldots, 0,1)^{t} \in \mathbb{R}^{2 n}$ to denote the vectors comprised of $n$ copies of the vectors $(1,0)^{t}$ or $(0,1)^{t}$, respectively. Note that by the construction the generalized adjacency matrices and block diagonal matrices satisfy

$$
D^{i j}(M) \mathbf{e}_{k}=A^{i j}(M) \mathbf{e}_{k} \quad \forall M \in\left\{\mathrm{Id}, \mathbf{x x}^{t}, \mathbf{y} \mathbf{y}^{t}, \mathbf{z z}^{t}\right\} \forall k \in\{1,2\} .
$$


Note also that an arbitrary unit vector $\mathbf{v} \in \mathbb{R}^{2 N}$ that is orthogonal to all vectors of the form $\mathbf{v}_{c}:=(\mathbf{w}, \ldots, \mathbf{w})^{t}$, where $\mathbf{w} \in \mathbb{R}^{2}$ is arbitrary, decomposes as

$$
\mathbf{v}=\frac{\alpha_{1}}{\sqrt{6 n}}\left(\begin{array}{c}
2 \mathbf{e}_{1} \\
-\mathbf{e}_{1} \\
-\mathbf{e}_{1}
\end{array}\right)+\frac{\alpha_{2}}{\sqrt{2 n}}\left(\begin{array}{c}
\mathbf{0} \\
\mathbf{e}_{1} \\
-\mathbf{e}_{1}
\end{array}\right)+\frac{\beta_{1}}{\sqrt{6 n}}\left(\begin{array}{c}
2 \mathbf{e}_{2} \\
-\mathbf{e}_{2} \\
-\mathbf{e}_{2}
\end{array}\right)+\frac{\beta_{2}}{\sqrt{2 n}}\left(\begin{array}{r}
\mathbf{0} \\
\mathbf{e}_{2} \\
-\mathbf{e}_{2}
\end{array}\right)+\gamma\left(\begin{array}{l}
\mathbf{w}_{1} \\
\mathbf{w}_{2} \\
\mathbf{w}_{3}
\end{array}\right)
$$

where $\alpha_{1}^{2}+\alpha_{2}^{2}+\beta_{1}^{2}+\beta_{2}^{2}+\gamma^{2}=1$ and each $\mathbf{w}_{k} \in \mathbb{R}^{2 n}$ satisfies

$$
\left\langle\mathbf{w}_{k}, \mathbf{e}_{1}\right\rangle=\left\langle\mathbf{w}_{k}, \mathbf{e}_{2}\right\rangle=0 .
$$

The eigenvector $\mathbf{v}_{r}$ of $L$ with eigenvalue zero that arises due to rotation invariance satisfies

$$
\mathbf{v}_{r}=\frac{1}{2}\left(\begin{array}{c}
2 \mathbf{e}_{2} \\
-\mathbf{e}_{2} \\
-\mathbf{e}_{2}
\end{array}\right)+\frac{\sqrt{3}}{2}\left(\begin{array}{c}
\mathbf{0} \\
-\mathbf{e}_{1} \\
\mathbf{e}_{1}
\end{array}\right)
$$

so that enforcing $\left\langle\mathbf{v}, \mathbf{v}_{r}\right\rangle=0$ for any $\mathbf{v}$ of the form (38) imposes the additional relation $\beta_{1}=\alpha_{2}$ on the coefficients. Thus we can write an arbitrary $\mathbf{v} \in V^{\perp}$ with $\|\mathbf{v}\|=1$ as

$$
\begin{aligned}
& \mathbf{v}=\frac{\alpha_{1}}{\sqrt{6 n}}\left(\begin{array}{c}
2 \mathbf{e}_{1} \\
-\mathbf{e}_{1} \\
-\mathbf{e}_{1}
\end{array}\right)+\frac{\beta_{1}}{\sqrt{2 n}}\left(\begin{array}{c}
\mathbf{0} \\
\mathbf{e}_{1} \\
-\mathbf{e}_{1}
\end{array}\right)+\frac{\beta_{1}}{\sqrt{6 n}}\left(\begin{array}{c}
2 \mathbf{e}_{2} \\
-\mathbf{e}_{2} \\
-\mathbf{e}_{2}
\end{array}\right)+\frac{\beta_{2}}{\sqrt{2 n}}\left(\begin{array}{c}
\mathbf{0} \\
\mathbf{e}_{2} \\
-\mathbf{e}_{2}
\end{array}\right)+\gamma\left(\begin{array}{l}
\mathbf{w}_{1} \\
\mathbf{w}_{2} \\
\mathbf{w}_{3}
\end{array}\right) \\
& \mathbf{v}:=\alpha_{1} \mathbf{v}_{1}+\beta_{1} \mathbf{v}_{2}+\beta_{2} \mathbf{v}_{3}+\gamma \mathbf{v}_{4}, \quad \alpha_{1}^{2}+2 \beta_{1}^{2}+\beta_{2}^{2}+\gamma^{2}=1 .
\end{aligned}
$$

We therefore wish to characterize when $\langle\mathbf{v}, L \mathbf{v}\rangle<0$ for any vector $\mathbf{v}$ satisfying (39), i.e. those vectors in the subspace $V^{\perp}=\operatorname{span}\left(\mathbf{v}_{c}, \mathbf{v}_{r}\right)^{\perp}$ with norm one.

We may now follow the proof of lemma 3.1 to extract the dominant components of $\langle\mathbf{v}, L \mathbf{v}\rangle$. Write $L=\kappa L(\mathrm{Id})-\tilde{L}$ for

$$
\tilde{L}:=L\left(\mathbf{x x}^{t}\right)+L\left(\mathbf{y} \mathbf{y}^{t}\right)+L\left(\mathbf{z z}^{t}\right),
$$

and note that (37) implies

$$
L(\mathrm{Id}) \mathbf{v}=L(\mathrm{Id}) \mathbf{v}_{4} .
$$

After some simplification, this yields as a consequence that

$$
\langle\mathbf{v}, L \mathbf{v}\rangle=\gamma^{2}\left\langle\mathbf{v}_{4}, L \mathbf{v}_{4}\right\rangle-\left\langle\alpha_{1} \mathbf{v}_{1}+\beta_{1} \mathbf{v}_{2}+\beta_{2} \mathbf{v}_{3}, \tilde{L}\left(\alpha_{1} \mathbf{v}_{1}+\beta_{1} \mathbf{v}_{2}+\beta_{2} \mathbf{v}_{3}\right)\right\rangle-2 \gamma\left\langle\alpha_{1} \mathbf{v}_{1}+\beta_{1} \mathbf{v}_{2}+\beta_{2} \mathbf{v}_{3}, \tilde{L} \mathbf{v}_{4}\right\rangle .
$$

Due to (37), a simple computation demonstrates that

$$
\sqrt{6 n}\left\langle\mathbf{v}_{1}, \tilde{L} \mathbf{v}_{4}\right\rangle=3\left\langle\left(A^{12}\left(\mathbf{x} \mathbf{x}^{t}\right)+A^{13}\left(\mathbf{y} \mathbf{y}^{t}\right)\right) \mathbf{e}_{1}, \mathbf{w}_{1}\right\rangle-3\left\langle A^{21}\left(\mathbf{x x}^{t}\right) \mathbf{e}_{1}, \mathbf{w}_{2}\right\rangle-3\left\langle A^{31}\left(\mathbf{x x}^{t}\right) \mathbf{e}_{1}, \mathbf{w}_{3}\right\rangle .
$$

Now realize that if $\tilde{\mathbf{w}}=\left(\mathbf{w}_{1}, \mathbf{0}, \mathbf{0}\right)^{t}$ and $\tilde{\mathbf{e}_{1}}=\left(\mathbf{0}, \mathbf{e}_{1}, \mathbf{0}\right)^{t}$ then asymptotically almost surely it holds that

$$
\left\langle\mathbf{w}_{1}, A^{12}\left(\mathbf{x x}^{t}\right) \mathbf{e}_{1}\right\rangle=\left\langle\tilde{\mathbf{w}}, A\left(\mathbf{x x}^{t}\right) \tilde{\mathbf{e}_{1}}\right\rangle=\sqrt{N} O(\sqrt{N p})
$$

uniformly for $\mathbf{v} \in V^{\perp}$. The last statement follows as $\tilde{\mathbf{w}} \in \mathcal{S}_{0}^{n d}$, so theorem 2.2 applies. In a similar fashion we have that asymptotically almost surely

$$
\left\langle A^{13}\left(\mathbf{y} \mathbf{y}^{t}\right) \mathbf{e}_{1}, \mathbf{w}_{1}\right\rangle=\sqrt{N} O(\sqrt{N p}),\left\langle A^{21}\left(\mathbf{x x}^{t}\right) \mathbf{e}_{1}, \mathbf{w}_{2}\right\rangle=\sqrt{N} O(\sqrt{N p})\left\langle A^{31}\left(\mathbf{y y}^{t}\right) \mathbf{e}_{1}, \mathbf{w}_{3}\right\rangle=\sqrt{N} O(\sqrt{N p})
$$

uniformly for $\mathbf{v} \in V^{\perp}$, so that $\left\langle\mathbf{v}_{1}, \tilde{L} \mathbf{v}_{4}\right\rangle=O(\sqrt{N p})$ asymptotically almost surely as well. Applying this argument twice more, with $\mathbf{v}_{2}$ and $\mathbf{v}_{3}$ in place of $\mathbf{v}_{1}$, suffices to demonstrate that

$$
\begin{aligned}
& \left\langle\mathbf{v}_{2}, \tilde{L} \mathbf{v}_{4}\right\rangle=O(\sqrt{N p}), \quad\left\langle\mathbf{v}_{3}, \tilde{L} \mathbf{v}_{4}\right\rangle=O(\sqrt{N p}), \quad \max _{\mathbf{v} \in V^{\perp}:\|\mathbf{v}\|=1}\langle\mathbf{v}, L \mathbf{v}\rangle= \\
& \left(\max _{\mathbf{v} \in V^{\perp}:\|\mathbf{v}\|=1} \gamma^{2}\left\langle\mathbf{v}_{4}, L \mathbf{v}_{4}\right\rangle-\left\langle\alpha_{1} \mathbf{v}_{1}+\beta_{1} \mathbf{v}_{2}+\beta_{2} \mathbf{v}_{3}, \tilde{L}\left(\alpha_{1} \mathbf{v}_{1}+\beta_{1} \mathbf{v}_{2}+\beta_{2} \mathbf{v}_{3}\right)\right\rangle\right)+O(\sqrt{N p}),
\end{aligned}
$$


asymptotically almost surely, where the last line follows due to the fact that $\max \left\{\left|\alpha_{1}\right|,\left|\beta_{1}\right|,\left|\beta_{2}\right|,|\gamma|\right\} \leq 1$ from the normalization requirement $\alpha_{1}^{2}+2 \beta_{1}^{2}+\beta_{2}^{2}+\gamma^{2}=1$.

We now turn to the second term. To write this quantity in a more tractible fashion, we first appeal to the following set of identities that follow by direct computation from the definitions of $\mathbf{x}, \mathbf{y}, \mathbf{z}$, the relation (37) and the construction of the generalized adjacency matrices-

$$
\begin{array}{llll}
\sqrt{3} A^{12}\left(\mathbf{x x}^{t}\right) \mathbf{e}_{2}+A^{12}\left(\mathbf{x x}^{t}\right) \mathbf{e}_{1}=\mathbf{0}, & \sqrt{3} A^{13}\left(\mathbf{y} \mathbf{y}^{t}\right) \mathbf{e}_{2}-A^{13}\left(\mathbf{y} \mathbf{y}^{t}\right) \mathbf{e}_{1}=\mathbf{0}, & A^{23}\left(\mathbf{z z}^{t}\right) \mathbf{e}_{1}=\mathbf{0} \\
\sqrt{3} A^{21}\left(\mathbf{x x}^{t}\right) \mathbf{e}_{2}+A^{21}\left(\mathbf{x x}^{t}\right) \mathbf{e}_{1}=\mathbf{0}, & \sqrt{3} A^{31}\left(\mathbf{y} \mathbf{y}^{t}\right) \mathbf{e}_{2}-A^{31}\left(\mathbf{y} \mathbf{y}^{t}\right) \mathbf{e}_{1}=\mathbf{0}, & A^{32}\left(\mathbf{z z}^{t}\right) \mathbf{e}_{1}=\mathbf{0} .
\end{array}
$$

These identities then allow us to simplify the action of $\tilde{L}$ on each of $\mathbf{v}_{1}, \mathbf{v}_{2}, \mathbf{v}_{3}$, in that we have

$$
\begin{aligned}
& \sqrt{6 n} \tilde{L} \mathbf{v}_{1}=3\left(A^{12}\left(\mathbf{x} \mathbf{x}^{t}\right) \mathbf{e}_{1}+A^{13}\left(\mathbf{y} \mathbf{y}^{t}\right) \mathbf{e}_{1},-A^{21}\left(\mathbf{x} \mathbf{x}^{t}\right) \mathbf{e}_{1},-A^{31}\left(\mathbf{y} \mathbf{y}^{t}\right) \mathbf{e}_{1}\right)^{t} \\
& \sqrt{n} \tilde{L} \mathbf{v}_{2}=\sqrt{2}\left(A^{13}\left(\mathbf{y y}^{t}\right) \mathbf{e}_{1}-A^{12}\left(\mathbf{x x}^{t}\right) \mathbf{e}_{1}, A^{21}\left(\mathbf{x} \mathbf{x}^{t}\right) \mathbf{e}_{1},-A^{31}\left(\mathbf{y} \mathbf{y}^{t}\right) \mathbf{e}_{1}\right)^{t} \\
& \sqrt{2 n} \tilde{L} \mathbf{v}_{3}=\left(A^{13}\left(\mathbf{y} \mathbf{y}^{t}\right) \mathbf{e}_{2}-A^{12}\left(\mathbf{x} \mathbf{x}^{t}\right) \mathbf{e}_{2}, A^{21}\left(\mathbf{x} \mathbf{x}^{t}\right) \mathbf{e}_{2}+2 A^{23}\left(\mathbf{z z}^{t}\right) \mathbf{e}_{2},-A^{31}\left(\mathbf{y} \mathbf{y}^{t}\right) \mathbf{e}_{2}-2 A^{32}\left(\mathbf{z z}^{t}\right) \mathbf{e}_{2}\right)^{t}
\end{aligned}
$$

These formulae, when combined with the previous identities, allow us to compute each possible combination of $\left\langle\mathbf{v}_{i}, \tilde{L} \mathbf{v}_{j}\right\rangle$ solely in terms of easily estimated, element-wise sums of the adjacency matrix $E$ of the underlying random graph. Specifically, we have that

$$
\begin{aligned}
& \frac{2 n}{3}\left\langle\mathbf{v}_{1}, \tilde{L} \mathbf{v}_{1}\right\rangle=\frac{n}{2}\left\langle\mathbf{v}_{2}, \tilde{L} \mathbf{v}_{2}\right\rangle=2 n\left\langle\mathbf{v}_{1}, \tilde{L} \mathbf{v}_{3}\right\rangle=\left\langle\mathbf{e}_{1}, A^{12}\left(\mathbf{x} \mathbf{x}^{t}\right) \mathbf{e}_{1}\right\rangle+\left\langle\mathbf{e}_{1}, A^{13}\left(\mathbf{y y}^{t}\right) \mathbf{e}_{1}\right\rangle \\
& \frac{n}{\sqrt{3}}\left\langle\mathbf{v}_{1}, \tilde{L} \mathbf{v}_{2}\right\rangle=\sqrt{3} n\left\langle\mathbf{v}_{3}, \tilde{L} \mathbf{v}_{2}\right\rangle=\left\langle\mathbf{e}_{1}, A^{13}\left(\mathbf{y} \mathbf{y}^{t}\right) \mathbf{e}_{1}\right\rangle-\left\langle\mathbf{e}_{1}, A^{12}\left(\mathbf{x} \mathbf{x}^{t}\right) \mathbf{e}_{1}\right\rangle \\
& 2 n\left\langle\mathbf{v}_{3}, \tilde{L} \mathbf{v}_{3}\right\rangle=4\left\langle\mathbf{e}_{2}, A^{23}\left(\mathbf{z} \mathbf{z}^{t}\right) \mathbf{e}_{2}\right\rangle+\left\langle\mathbf{e}_{2}, A^{12}\left(\mathbf{x} \mathbf{x}^{t}\right) \mathbf{e}_{2}\right\rangle+\left\langle\mathbf{e}_{2}, A^{13}\left(\mathbf{y} \mathbf{y}^{t}\right) \mathbf{e}_{2}\right\rangle .
\end{aligned}
$$

Note that the construction of the generalized adjacency matrices $A\left(\mathbf{x x}^{t}\right), A\left(\mathbf{y} \mathbf{y}^{t}\right)$ and $A\left(\mathbf{z z}^{t}\right)$ implies that

$$
\begin{aligned}
\left\langle\mathbf{e}_{1}, A^{12}\left(\mathbf{x} \mathbf{x}^{t}\right) \mathbf{e}_{1}\right\rangle & =\left(\sum_{i=1}^{n} \sum_{j=n+1}^{2 n} e_{i j}\right)\left(\mathbf{x}^{t} \mathbf{e}_{1}\right)^{2} \quad\left\langle\mathbf{e}_{2}, A^{12}\left(\mathbf{x} \mathbf{x}^{t}\right) \mathbf{e}_{2}\right\rangle=\left(\sum_{i=1}^{n} \sum_{j=n+1}^{2 n} e_{i j}\right)\left(\mathbf{x}^{t} \mathbf{e}_{2}\right)^{2} \\
\left\langle\mathbf{e}_{1}, A^{13}\left(\mathbf{y} \mathbf{y}^{t}\right) \mathbf{e}_{1}\right\rangle & =\left(\sum_{i=1}^{n} \sum_{j=2 n+1}^{3 n} e_{i j}\right)\left(\mathbf{y}^{t} \mathbf{e}_{1}\right)^{2} \quad\left\langle\mathbf{e}_{2}, A^{13}\left(\mathbf{y} \mathbf{y}^{t}\right) \mathbf{e}_{2}\right\rangle=\left(\sum_{i=1}^{n} \sum_{j=2 n+1}^{3 n} e_{i j}\right)\left(\mathbf{y}^{t} \mathbf{e}_{2}\right)^{2} \\
\left\langle\mathbf{e}_{2}, A^{23}\left(\mathbf{z z}^{t}\right) \mathbf{e}_{2}\right\rangle & =\left(\sum_{i=n+1}^{2 n} \sum_{j=2 n+1}^{3 n} e_{i j}\right)\left(\mathbf{z}^{t} \mathbf{e}_{2}\right)^{2},
\end{aligned}
$$

where $e_{i j}$ denote the edges of the underlying random graph. Following lemma 3.1, if $n p=\Theta(\log n)$ then standard concentration of measure arguments (i.e. the Chernoff bound) imply that

$$
\sum_{i=1}^{n} \sum_{j=n+1}^{2 n} e_{i j}=n(n p+O(\sqrt{N p}))
$$

with probability at least $1-2 \mathrm{e}^{-n / 4}$. Analogous results hold for the remaining edge sums in (43). We now substitute this fact, along with the facts that $\left(\mathbf{x}^{t} \mathbf{e}_{1}\right)^{2}=\left(\mathbf{y}^{t} \mathbf{e}_{1}\right)^{2}=3 / 4,\left(\mathbf{x}^{t} \mathbf{e}_{1}\right)^{2}=\left(\mathbf{y}^{t} \mathbf{e}_{1}\right)^{2}=1 / 4$ and $\left(\mathbf{z}^{t} \mathbf{e}_{2}\right)^{2}=1$, into (42) to conclude that

$$
\left\langle\alpha_{1} \mathbf{v}_{1}+\beta_{1} \mathbf{v}_{2}+\beta_{2} \mathbf{v}_{3}, \tilde{L}\left(\alpha_{1} \mathbf{v}_{1}+\beta_{1} \mathbf{v}_{2}+\beta_{2} \mathbf{v}_{3}\right)\right\rangle=-\left[\frac{3}{2}\left(\alpha_{1}^{2}+2 \beta_{1}^{2}+\beta_{2}^{2}\right)+\frac{3}{4}\left(\alpha_{1}+\beta_{2}\right)^{2}\right] n p+O(\sqrt{N p}) .
$$

This estimate holds with probability at least $1-c \mathrm{e}^{-n / 4}$ uniformly for $\mathbf{v} \in V^{\perp}$, so that the subsequent estimate

$$
\begin{aligned}
& \max _{\mathbf{v} \in V^{\perp}:\|\mathbf{v}\|=1} \gamma^{2}\left\langle\mathbf{v}_{4}, L \mathbf{v}_{4}\right\rangle-\left\langle\alpha_{1} \mathbf{v}_{1}+\beta_{1} \mathbf{v}_{2}+\beta_{2} \mathbf{v}_{3}, \tilde{L}\left(\alpha_{1} \mathbf{v}_{1}+\beta_{1} \mathbf{v}_{2}+\beta_{2} \mathbf{v}_{3}\right)\right\rangle= \\
& \left(\max _{\mathbf{v} \in V^{\perp}:\|\mathbf{v}\|=1} \gamma^{2}\left\langle\mathbf{v}_{4}, L \mathbf{v}_{4}\right\rangle-\left[\frac{3}{2}\left(\alpha_{1}^{2}+2 \beta_{1}^{2}+\beta_{2}^{2}\right)+\frac{3}{4}\left(\alpha_{1}+\beta_{2}\right)^{2}\right] n p\right)+O(\sqrt{N p}) .
\end{aligned}
$$


also holds with at least this probability.

It remains to estimate $\left\langle\mathbf{v}_{4}, L \mathbf{v}_{4}\right\rangle$, which we decompose as

$$
\left\langle\mathbf{v}_{4}, L \mathbf{v}_{4}\right\rangle=\left\langle\mathbf{v}_{4}, D_{L} \mathbf{v}_{4}\right\rangle-\left\langle\mathbf{v}_{4}, B_{L} \mathbf{v}_{4}\right\rangle .
$$

Here $D_{L}:=\kappa D(\mathrm{Id})-\left(D\left(\mathbf{x x}^{t}\right)+D\left(\mathbf{y} \mathbf{y}^{t}\right)+D\left(\mathbf{z z}^{t}\right)\right)$ denotes the block-diagonal component of $L$ and $B_{L}:=$ $-\kappa B(\mathrm{Id})+B\left(\mathbf{x x}^{t}\right)+B\left(\mathbf{y} \mathbf{y}^{t}\right)+B\left(\mathbf{z z}^{t}\right)$ denotes the off-diagonal component. As in the one dimensional case, we may reduce our analysis of the stability of the two-dimensional compromise solution to a study of when the largest eigenvalue $\lambda_{1}\left(D_{L}\right)$ of $D_{L}$ is sufficiently negative. Specifically, suppose there exists a $c_{1}>0$ so that $\lambda_{1}\left(D_{L}\right) \leq-c_{1} N p$ asymptotically almost surely. Then as $\left\|\mathbf{v}_{4}\right\|=1$ it follows that

$$
\left\langle\mathbf{v}_{4}, L \mathbf{v}_{4}\right\rangle \leq-c_{1} N p-\left\langle\mathbf{v}_{4}, B_{L} \mathbf{v}_{4}\right\rangle .
$$

In much the same manner as we arrived at (41), it follows from theorem 2.2 that

$$
\left\langle\mathbf{v}_{4}, B_{L} \mathbf{v}_{4}\right\rangle=O(\sqrt{N p})
$$

uniformly for $\mathbf{v} \in V^{\perp}$. Combining this with the previous reductions, if $N p=\Theta(\log N)$ and $\lambda_{1}\left(D_{L}\right) \leq-c_{1} N p$ asymptotically almost surely then

$$
\max _{\mathbf{v} \in V^{\perp}:\|\mathbf{v}\|=1}\langle\mathbf{v}, L \mathbf{v}\rangle \leq-\min \left\{c_{1}, \frac{1}{2}\right\} N p+O(\sqrt{N p})<0
$$

asymptotically almost surely as well. In other words, the two-dimensional simplex configuration is stable.

\subsection{Estimating the Diagonal}

With these reductions in place, the procedure for determining the threshold follows the program outlined in the one dimensional case. Given any of the $2 \times 2$ blocks $D_{i i}$ that constitute the diagonal component $D$ of the stability matrix, define

$$
f_{n}\left(p, \kappa, c_{1}\right):=\mathbb{P}\left(\lambda_{1}\left(D_{i i}\right) \geq c_{1} n p\right) .
$$

We may determine the critical probability $p_{c}$ from the relation

$$
f_{n}\left(p_{c}, \kappa, 0\right)=c_{2}(n) \Theta\left(\frac{1}{n}\right),
$$

where $c_{2}(n)$ denotes a function of $n$ that grows (or decays) more slowly than any power. This again amounts to a computation involving independent binomial distributions. If $p=(1+\epsilon) p_{c}$ then there exists a $c_{1}<0$ so that

$$
f_{n}\left(p, \kappa, c_{1}\right)=O\left(n^{-1-\epsilon / 2}\right),
$$

and this implies stability asymptotically almost surely by the previous reductions and the union bound. Conversely, when $p=(1-\epsilon) p_{c}$ then

$$
f_{n}\left(p, \kappa, c_{1}\right) \geq c^{\prime} n^{-1+\epsilon / 2}
$$

for some $c_{1}>0$, and this implies instability asymptotically almost surely.

The principle that underlies the calculation of $p_{c}$ is straightforward. Nevertheless, this computation can prove quite technical as the one dimensional case shows. For the sake of brevity, we shall content ourselves with an easily established upper bound on $p_{c}$ for now and leave a full calculation of the threshold for future work. For $1 \leq i \leq n$ note that

$$
\begin{aligned}
D_{i i} & =\kappa\left(\sum_{i=1}^{n} e_{i j}\right) \mathrm{Id}-\left(\sum_{i=n+1}^{2 n} e_{i j}\right) \mathbf{x} \mathbf{x}^{t}-\left(\sum_{i=2 n+1}^{3 n} e_{i j}\right) \mathbf{y y}^{t}, \\
\mathbb{E}\left(D_{i i}\right) & =n p\left(\kappa \mathrm{Id}-\mathbf{x x}^{t}-\mathbf{y y}^{t}\right) .
\end{aligned}
$$


The definitions of $\mathbf{x}$ and $\mathbf{y}$ show that $(\mathbf{x}-\mathbf{y})$ yields the eigenvector of $\mathbb{E}\left(D_{i i}\right)$ with largest eigenvalue along with its value

$$
\lambda_{1}\left(\mathbb{E}\left(D_{i i}\right)\right)=\left(\kappa-\frac{1}{2}\right) n p .
$$

When $n p \geq(1+\epsilon) \log n$ for some $\epsilon>0$, the Chernoff bound yields

$$
\left|\sum_{i=1}^{n} e_{i j}-n p\right| \leq 2 \sqrt{(1+\epsilon) n p \log n}
$$

with probability at least $1-2 n^{-(1+\epsilon)}$. As a consequence, the union bound and the triangle inequality combine to show that with probability at least $1-O\left(n^{-(1+\epsilon)}\right)$ the estimate

$$
\lambda_{1}\left(D_{i i}\right) \leq n p\left(\kappa-\frac{1}{2}+6 \sqrt{\log n(1+\epsilon) / n p}\right)
$$

holds. For $0<\kappa<1 / 2$ fixed and

$$
n p>\frac{36(1+\epsilon) \log n}{(\kappa-1 / 2)^{2}}
$$

it follows that there exists $c_{1}>0$ so that $\lambda_{1}\left(D_{i i}\right) \leq-c_{1} n p$ with probability at least $1-O\left(n^{-(1+\epsilon)}\right)$. Of course, the same estimate holds for $\lambda_{1}\left(D_{i i}\right)$ when $n+1 \leq i \leq N$ as well. The union bound then demonstrates that

$$
\lambda_{1}(D) \leq-c_{1} n p
$$

with probability at least $1-O\left(n^{-\epsilon}\right)$, which suffices to yield stability asymptotically almost surely by the previous reductions. We may summarize the preceeding in the following theorem:

Theorem 4.2. Fix $0<\kappa<1 / 2$ and $\epsilon>0$. If $n p(\kappa-1 / 2)^{2}>36(1+\epsilon) \log n$ then the two dimensional compromise solution, with $n=N / 3$ individuals in each group, is stable asymptotically almost surely. That is, (36) holds with probability approaching one as $n \rightarrow \infty$.

This theorem implies that $n p=O(\log n)$ suffices to guarantee stability of the two-dimensional simplex asymptotically almost surely. Using a connectivity-based argument similar to that used in [49], we may conclude that stability asymptotically almost surely necessitates $n p \geq c \log n$ for some constant $c>0$ as well. In other words, the stability threshold for the two-dimensional simplex configuration exhibits the same critical scaling $n p \propto \log n$ as the one-dimensional case.

\section{Conclusion}

This paper analyzes the behavior of a large system of interacting particles whose interaction structure is dictated by an Erdős-Rényi random graph. Specifically, we proved theorems that yield stability or instability for two types of simplex equilibria as the number of particles becomes infinite. For the one-dimensional simplex equilibria we rigorously established a conjecture first formulated in [49], i.e. an explicit formula for the critical probability above which stability holds asymptotically almost surely. We also established that the threshold for two-dimensional simplex equilibria exhibits the same critical scaling, with respect to the number of particles, as the one-dimensional case. Moreover, these same arguments reduce complicated stability estimates to a more straightforward estimation of weighted differences of binomial distributions. This reduction should allow for the calculation of an explicit threshold in the two dimensional in a manner analogous to the one-dimensional setting. We leave an investigation of this threshold for future work, however. We also leave a study of arbitrary $d$-dimensional simplex equilibria for future work, although in principle our two-dimensional arguments generalize in a straightforward way to handle simplices in arbitrary dimensions. Our present work does not, however, shed light on the more complicated, random structures that do not lie in equilibrium under all choices of random graphs. Studying these structures also presents an interesting open problem, but its resolution likely requires different techniques than we employ here. 


\section{Acknowledgements}

JvB and ALB acknowledge funding from NSF grant DMS-0914856 and AFOSR MURI grant FA9550-101-0569. JvB also acknowledges funding from NSF grant DMS-1312344. The research of B. Sudakov is supported in part by AFOSR MURI grant FA9550-10-1-0569, by a USA-Israeli BSF grant and by SNSF grant 200021-149111.

\section{A Estimates for Generalized Adjacency Matrices}

Given a random graph drawn from $\mathcal{G}(n, p)$, let $A \in M_{n d \times n d}(\mathbb{R})$ denote an $n d \times n d$ generalized adjacency matrix using any symmetric matrix $M \in \mathbb{M}_{d \times d}(\mathbb{R})$ as a sub-block. In other words, if $E$ denotes the adjacency matrix of the graph then we have

$$
A=E \otimes M
$$

for $\otimes$ denoting the Kronecker product. Let $M_{j k}=M_{k j}, 1 \leq j \leq n, j \leq k \leq n$ denote the i.i.d. matrixvalued random variables corresponding to the edges in the graph, so that $\mathbb{E}\left(M_{j k}\right)=p M$. For a given a vector $\mathbf{x} \in \mathbb{R}^{n d}$ consider the partition $\mathbf{x}=\left(\mathbf{x}_{1}, \ldots, \mathbf{x}_{n}\right)^{t}$ for $\mathbf{x}_{i} \in \mathbb{R}^{d}$, and recall the "mean-zero" hypothesis

$$
\sum_{i=1}^{n} \mathbf{x}_{i}=\mathbf{0}
$$

If we denote the corresponding subset of the unit ball $\mathcal{S}^{\text {nd }} \subset \mathbb{R}^{n d}$ as

$$
\mathcal{S}_{0}^{n d}:=\left\{\mathbf{x}: \sum_{i} \mathbf{x}_{i}=\mathbf{0}, \quad \sum_{i}\left\|\mathbf{x}_{i}\right\|_{2}^{2} \leq 1\right\},
$$

our aim lies in proving the following generalization of the theorem due to [13]:

Theorem A.1. Let $\alpha$ and $c_{0}$ denote arbitrary positive constants. If $n p>c_{0} \log n$ then there exists a constant $c=c\left(\alpha, c_{0}, d,\|M\|_{2}\right)>0$ so that the estimate

$$
\max _{(\mathbf{x}, \mathbf{y}) \in \mathcal{S}_{0}^{n d} \times \mathcal{S}^{n d}}|\langle\mathbf{x}, A \mathbf{y}\rangle| \leq c \sqrt{n p}
$$

holds with probability at least $1-n^{-\alpha}$.

Note carefully that we only require one of $\mathbf{x}$ or $\mathbf{y}$ to satisfy the mean zero property (47). The proof of the theorem essentially reproduces the arguments of [13] by changing a few scalars to vectors and multiplications to inner products. The first ingredient is the following lemma:

Lemma A.2. Fix $(\mathbf{x}, \mathbf{y}) \in \mathcal{S}_{0}^{n d} \times \mathcal{S}^{n d}$ and let $\Lambda=\left\{(j, k):\left|\left\langle\mathbf{x}_{k}, M \mathbf{y}_{j}\right\rangle\right| \leq \sqrt{p / n}\right\}$. Then

$$
\left|\mathbb{E}\left(\sum_{(j, k) \in \Lambda}\left\langle\mathbf{x}_{k}, M_{k j} \mathbf{y}_{j}\right\rangle\right)\right| \leq\|M\|_{2}^{2} \sqrt{n p}
$$

Proof. As $\mathbf{x} \in \mathcal{S}_{0}^{\text {nd }}$ it follows that

$$
0=p \sum_{j, k}\left\langle\mathbf{x}_{k}, M \mathbf{y}_{j}\right\rangle=\mathbb{E}\left(\sum_{(j, k) \in \Lambda}\left\langle\mathbf{x}_{k}, M_{k j} \mathbf{y}_{j}\right\rangle\right)+p \sum_{(j, k) \in \Lambda^{c}}\left\langle\mathbf{x}_{k}, M \mathbf{y}_{j}\right\rangle .
$$

By definition, whenever $(j, k) \in \Lambda^{c}$ it follows that $\left|\left\langle\mathbf{x}_{k}, M \mathbf{y}_{j}\right\rangle\right|>\sqrt{p / n}$. Thus $p\left|\left\langle\mathbf{x}_{k}, M \mathbf{y}_{j}\right\rangle\right|<\sqrt{n p}\left|\left\langle\mathbf{x}_{k}, M \mathbf{y}_{j}\right\rangle\right|^{2} \leq$ $\sqrt{n p}|| M\left|\left\|_{2}^{2}|| \mathbf{x}_{k}\right\|_{2}^{2}\left\|\mathbf{y}_{j}\right\|_{2}^{2}\right.$ for any such $(j, k)$, where the last inequality follows from Cauchy-Schwarz. Combining these facts yields

$$
\left|\mathbb{E}\left(\sum_{(j, k) \in \Lambda}\left\langle\mathbf{x}_{k}, M_{k j} \mathbf{y}_{j}\right\rangle\right)\right|=p\left|\sum_{(j, k) \in \Lambda^{c}}\left\langle\mathbf{x}_{k}, M \mathbf{y}_{j}\right\rangle\right| \leq\|M\|_{2}^{2} \sqrt{n p} \sum_{j, k}\left\|\mathbf{x}_{k}\right\|_{2}^{2}\left\|\mathbf{y}_{j}\right\|_{2}^{2}=\|M\|_{2}^{2} \sqrt{n p}\|\mathbf{x}\|_{2}^{2}\|\mathbf{y}\|_{2}^{2}
$$

as desired. 
For a fixed $(\mathbf{x}, \mathbf{y}) \in \mathcal{S}_{0}^{n d} \times \mathcal{S}^{\text {nd }}$ let $S(\mathbf{x}, \mathbf{y}), L(\mathbf{x}, \mathbf{y}), U(\mathbf{x}, \mathbf{y})$ denote the random variables defined as

$$
S(\mathbf{x}, \mathbf{y}):=\sum_{(j, k) \in \Lambda}\left\langle\mathbf{x}_{j}, M_{j k} \mathbf{y}_{k}\right\rangle=\sum_{\substack{(j, k) \in \Lambda \\ j \leq k}}\left\langle\mathbf{x}_{j}, M_{j k} \mathbf{y}_{k}\right\rangle+\sum_{\substack{(j, k) \in \Lambda \\ j>k}}\left\langle\mathbf{x}_{j}, M_{j k} \mathbf{y}_{k}\right\rangle:=U(\mathbf{x}, \mathbf{y})+L(\mathbf{x}, \mathbf{y}) .
$$

Although dependencies exist between $L$ and $U$ due to the undirected graph, when considered in isolation each random variable is simply a sum of independent indicator random variables. Indeed, fix any ordering of the indices $\Lambda \cap\{j \leq k\}$ and write $U(\mathbf{x}, \mathbf{y})=\sum_{i} u_{i}$, where the sum ranges from one to the (deterministic) size of $\Lambda \cap\{j \leq k\}$, and note that each $u_{i}$ is either zero or $\left\langle\mathbf{x}_{j}, M \mathbf{y}_{k}\right\rangle$ with probability $(1-p)$ or $p$, respectively. Obviously for $L(\mathbf{x}, \mathbf{y})$ an analogous statement holds.

Note that $\left|u_{i}\right| \leq \sqrt{p / n}$ by definition of $\Lambda$, and that

$$
\sum_{i} \operatorname{Var}\left(u_{i}\right)=p(1-p) \sum_{\Lambda \cap\{j \leq k\}}\left|\left\langle\mathbf{x}_{j}, M \mathbf{y}_{k}\right\rangle\right|^{2} \leq p\|M\|_{2}^{2}\|\mathbf{x}\|_{2}^{2}\|\mathbf{y}\|_{2}^{2} \leq p\|M\|_{2}^{2} .
$$

By applying the Chernoff bound 2.1 to the random variables $\left(u_{i}-\mathbb{E}\left(u_{i}\right)\right) / 2 \sqrt{p / n}$ with the choices $\sigma^{2}=$ $n K|| M \|_{2}^{2} / 4$ and $\lambda^{2}=n K\|M\|_{2}^{2}$ these facts imply that for any $K \geq 1$ the estimate

$$
\mathbb{P}\left(|U(\mathbf{x}, \mathbf{y})-\mathbb{E}(U(\mathbf{x}, \mathbf{y}))| \geq K \sqrt{n p}\|M\|_{2}^{2}\right) \leq 2 \mathrm{e}^{-K n\|M\|_{2} / 4}
$$

holds. A similar argument shows that the same inequality holds for $L(\mathbf{x}, \mathbf{y})$ as well. As $S=L+U$, the triangle inequality and the union bound yield the estimate

$$
\mathbb{P}\left(|S(\mathbf{x}, \mathbf{y})-\mathbb{E}(S(\mathbf{x}, \mathbf{y}))| \geq 2 K \sqrt{n p}\|M\|_{2}^{2}\right) \leq 4 \mathrm{e}^{-K n\|M\|_{2} / 4} .
$$

Next, given $0<\delta<1$ define the finite grid

$$
T^{\delta}:=\left\{\mathbf{x} \in\left(\frac{\delta}{\sqrt{n}} \mathbb{Z}\right)^{n d}:\|\mathbf{x}\|_{2} \leq 1\right\}
$$

and its mean-zero variant

$$
T_{0}^{\delta}:=\left\{\mathbf{x} \in\left(\frac{\delta}{\sqrt{n}} \mathbb{Z}\right)^{n d}: \sum_{i} \mathbf{x}_{i}=0 \quad\|\mathbf{x}\|_{2} \leq 1\right\}
$$

The following lemma allows us to control the norm of $A$ on $\mathcal{S}_{0}^{n d}$ by controlling $\langle\mathbf{x}, A \mathbf{y}\rangle$ for pairs of vectors in the finite grid instead:

Lemma A.3. If $|\langle\mathbf{x}, A \mathbf{y}\rangle| \leq c$ for all $(\mathbf{x}, \mathbf{y}) \in T_{0}^{\delta} \times T^{\delta}$ then $|\langle\mathbf{x}, A \mathbf{y}\rangle| \leq \frac{c d}{(1-\delta)^{2}}$ for all $(\mathbf{x}, \mathbf{y}) \in \mathcal{S}_{0}^{n d} \times \mathcal{S}^{\text {nd }}$.

Proof. Let $\mathbf{z}=(1-\delta) \mathbf{x}, \mathbf{u}=(1-\delta) \mathbf{y}$ and note that

$$
\sum_{i} \mathbf{z}_{i}=\mathbf{0}
$$

Decompose $\mathbf{z}$ as $\mathbf{z}=\mathbf{z}_{1}+\cdots+\mathbf{z}_{d}$ where the non-zero components of $\mathbf{z}_{k}$ correspond to the $k^{\text {th }}$ equation in (51); that is, $\mathbf{z}_{k}$ contains the $k^{\text {th }},(d+k)^{\text {th }},(2 d+k)^{\text {th }}$, etc. components of $\mathbf{z}$ and has zeros elsewhere. As

$$
\|\mathbf{z}\|_{2}^{2}=\left\|\mathbf{z}_{1}\right\|^{2}+\cdots+\left\|\mathbf{z}_{d}\right\|^{2} \leq(1-\delta)^{2},
$$

the vector in $\mathbb{R}^{n}$ comprised of the non-zero locations of $\mathbf{z}_{k}$ has norm less than $(1-\delta)$ and entries that sum to zero. By lemma 2.3 of [13], each $\mathbf{z}_{k}$ is therefore a convex combination of points of $T_{0}^{\delta}$,

$$
\mathbf{z}_{k}=\sum_{j=1}^{J_{k}} \theta_{j}^{k} \mathbf{v}_{j}^{k}, \quad \theta_{j}^{k}>0, \quad \sum_{j=1}^{J_{k}} \theta_{j}^{k}=1 .
$$


Summing the $\mathbf{z}_{k}$ then shows that there exists an $N=J_{1}+\cdots+J_{d} \in \mathbb{N}, \theta_{l}>0$ and $\mathbf{v}_{l} \in T_{0}^{\delta}$ so that

$$
\mathbf{z}=\sum_{l=1}^{N} \theta_{l} \mathbf{v}_{l}, \quad \sum_{l=1}^{N} \theta_{l}=d .
$$

Lemma 2.3 of [13] also implies that $\mathbf{u}$ is a convex combination of points in $T^{\delta}$, so that there exist $M \in \mathbb{N}$, $\eta_{j}>0$ and $\mathbf{w}_{j} \in T^{\delta}$ so that

$$
\mathbf{u}=\sum_{j=1}^{M} \eta_{j} \mathbf{w}_{j}, \quad \sum_{j=1}^{M} \eta_{j}=1
$$

As a consequence,

$$
(1-\delta)^{2}|\langle\mathbf{x}, A \mathbf{y}\rangle|=|\langle\mathbf{z}, A \mathbf{u}\rangle|=\left|\sum_{l=1}^{N} \theta_{l} \sum_{j=1}^{M} \eta_{j}\left\langle\mathbf{v}_{l}, A \mathbf{w}_{j}\right\rangle\right| \leq c \sum_{l=1}^{N} \theta_{l} \sum_{j=1}^{M} \eta_{j}=c d .
$$

An estimate of the total number of points $\left|T^{\delta}\right|,\left|T_{0}^{\delta}\right|$ in the $\delta$-nets follows from a direct appeal to claim 2.9 of [13]. As $T_{0}^{\delta} \subset T^{\delta}$ and $\left|T^{\delta}\right| \leq \mathrm{e}^{c(n d)}$ for some constant $c$ that depends on $\delta$, which follows from claim 2.9 of [13], it follows that $\left|T_{0}^{\delta} \times T^{\delta}\right| \leq \mathrm{e}^{2 c(n d)}$ as well. Applying the union bound over $T_{0}^{\delta} \times T^{\delta}$, we may therefore summarize the preceeding in the following lemma:

Lemma A.4. Given any $c>0$ there exists $c^{\prime}>0$ so that with probability at least $1-\mathrm{e}^{-c n}$ the estimate

$$
\max _{(\mathbf{x}, \mathbf{y}) \in T_{0}^{\delta} \times T^{\delta}}|S(\mathbf{x}, \mathbf{y})| \leq c^{\prime} \sqrt{n p}
$$

holds.

It remains to estimate, for $(\mathbf{x}, \mathbf{y}) \in T_{0}^{\delta} \times T^{\delta}$, the remaining contribution

$$
H(\mathbf{x}, \mathbf{y}):=\langle\mathbf{x}, A \mathbf{y}\rangle-S(\mathbf{x}, \mathbf{y})=\sum_{(j, k) \in \Lambda^{c}}\left\langle\mathbf{x}_{j}, M_{j k} \mathbf{y}_{k}\right\rangle
$$

where we recall $\Lambda^{c}:=\left\{(j, k):\left|\left\langle\mathbf{x}_{j}, M \mathbf{y}_{k}\right\rangle\right|>\sqrt{p / n}\right\}$. From Cauchy-Schwarz it follows that

$$
|H(\mathbf{x}, \mathbf{y})| \leq\|M\|_{2} \sum_{(k, l) \in \Lambda^{c}}\left\|\mathbf{x}_{k}\right\|_{2}\left\|\mathbf{y}_{l}\right\|_{2} \mathbf{1}_{\left\{e_{k l}=1\right\}} .
$$

Here and in what follows, for an index subset $W$ the notation $\mathbf{1}_{W}$ denotes the indicator function. If we define the sets

$$
\begin{aligned}
X_{i} & :=\left\{k \in\{1 \ldots n\}: \frac{\delta}{\sqrt{n}} 2^{i-1} \leq\left\|\mathbf{x}_{k}\right\|_{2}<\frac{\delta}{\sqrt{n}} 2^{i}\right\} \\
Y_{i} & :=\left\{k \in\{1 \ldots n\}: \frac{\delta}{\sqrt{n}} 2^{i-1} \leq\left\|\mathbf{y}_{k}\right\|_{2}<\frac{\delta}{\sqrt{n}} 2^{i}\right\}
\end{aligned}
$$

and fix an edge $(k, l)$ corresponding to a non-zero term in the sum (53), then $k \in X_{i}$ and $l \in Y_{j}$ for some $(i, j)$ and an edge $(k, l)$ exists between these two vertex sets. Moreover, as $(k, l) \in \Lambda^{c}$ it follows that

$$
\sqrt{p / n}<\left|\left\langle\mathbf{x}_{k}, M \mathbf{y}_{l}\right\rangle\right| \leq \delta^{2}\|M\|_{2} 2^{i+j} / n \quad \Rightarrow \quad 2^{i} 2^{j}>\sqrt{p n}
$$

provided we take $\delta\|M\|_{2} \leq 1$. As a consequence,

$$
|H(\mathbf{x}, \mathbf{y})| \leq \delta^{2}\|M\|_{2} \sum_{\substack{i, j \\ 2^{i} 2^{j}>\sqrt{n p}}} \operatorname{edge}\left(X_{i}, Y_{j}\right) \frac{2^{i} 2^{j}}{n},
$$


where edge $\left(X_{i}, Y_{j}\right)$ denotes the number of edges between the sets.

To bound (54), we let $c_{1}$ denote an as-yet-undetermined constant and put

$$
W:=\left\{(i, j): 2^{i} 2^{j}>\sqrt{n p}, \max \left\{\left|X_{i}\right|,\left|Y_{j}\right|\right\}>(n / \mathrm{e})\right\},
$$

then decompose the sum further as

$$
\sum_{\substack{i, j \\ 2^{i} 2^{j}>\sqrt{n p}}} \operatorname{edge}\left(X_{i}, Y_{j}\right) \frac{2^{i} 2^{j}}{n}=\sum_{W} \operatorname{edge}\left(X_{i}, Y_{j}\right) \frac{2^{i} 2^{j}}{n}+\sum_{W^{c}} \operatorname{edge}\left(X_{i}, Y_{j}\right) \frac{2^{i} 2^{j}}{n} .
$$

Assuming that each vertex has degree bounded by $c_{1} n p$ (c.f. lemma A.5) then $\operatorname{edge}\left(X_{i}, Y_{j}\right) \leq c_{1} n p \min \left\{\left|X_{i}\right|,\left|Y_{j}\right|\right\}$. Thus the first term is bounded by

$$
\sum_{W} \operatorname{edge}\left(X_{i}, Y_{j}\right) \frac{2^{i} 2^{j}}{n} \leq c_{1} \mathrm{e} p \sum_{W} \min \left\{\left|X_{i}\right|,\left|Y_{j}\right|\right\} \max \left\{\left|X_{i}\right|,\left|Y_{j}\right|\right\} \frac{2^{i} 2^{j}}{n} \leq c_{1} \mathrm{e} \sqrt{n p} \sum_{i, j} 2^{2 i}\left|X_{i}\right| 2^{2 j}\left|Y_{j}\right| n^{-2}
$$

Noting that

$$
\sum_{i} \frac{\left|X_{i}\right| 2^{2 i}}{n} \leq 4 \delta^{-2}\|\mathbf{x}\|^{2} \quad \sum_{j} \frac{\left|Y_{j}\right| 2^{2 j}}{n} \leq 4 \delta^{-2}\|\mathbf{y}\|^{2}
$$

then shows that the first term is $O(\sqrt{n p})$ provided the bounded degree property holds. Fortunately, if $n p>c_{0} \log n$ for any $c_{0}>0$, this property holds with probability at least $1-n^{-\alpha}$ for any $\alpha>0$ provided $c_{1}$ is sufficiently large:

Lemma A.5. (Bounded Degree) Let $p \geq c_{0} \log n / n$ for any $c_{0}>0$ and $\operatorname{deg}_{1}, \ldots, \operatorname{deg}_{n}$ denote the vertex degrees of an Erdös-Rényi random graph $\mathcal{G}(n, p)$. For any $\alpha>0$ there exists a $c_{1}=c_{1}\left(c_{0}, \alpha\right)>2$ so that

$$
\mathbb{P}\left(\max _{i} \operatorname{deg}_{i}>c_{1} n p\right) \leq 2 n^{-\alpha} .
$$

Proof. Write $\operatorname{deg}_{i}=\sum_{j} e_{i j}$ where $e_{i j}$ denote the edges of the graph. As $\mathbb{E}\left(\operatorname{deg}_{i}\right)=n p$ and $\operatorname{Var}\left(\operatorname{deg}_{i}\right)=$ $n p(1-p) \leq\left(c_{1}-1\right) n p$ it follows from the Chernoff bound and the union bound that

$$
\mathbb{P}\left(\left|\operatorname{deg}_{i}-n p\right|>\lambda \sqrt{\left(c_{1}-1\right) n p}\right) \leq 2 \mathrm{e}^{-\lambda^{2} / 4} \Rightarrow \mathbb{P}\left(\max _{i}\left|\operatorname{deg}_{i}-n p\right|>\lambda \sqrt{\left(c_{1}-1\right) n p}\right) \leq 2 \mathrm{e}^{-\lambda^{2} / 4+\log n} .
$$

The choice $\lambda=\sqrt{\left(c_{1}-1\right) n p}$ yields

$$
\mathbb{P}\left(\max _{i}\left|\operatorname{deg}_{i}-n p\right|>\left(c_{1}-1\right) n p\right) \leq 2 \mathrm{e}^{-n p\left(c_{1}-1\right) / 4+\log n} \leq 2 e^{\log n\left(1-c_{0}\left(c_{1}-1\right) / 4\right)} .
$$

Taking $c_{1}$ sufficiently large gives the desired result.

Therefore with probability at least $1-n^{-\alpha}$, the first term in $(55)$ is $O(\sqrt{n p})$ uniformly for $(\mathbf{x}, \mathbf{y}) \in T_{0}^{\delta} \times T^{\delta}$ as long as the bounded degree property holds. It remains to handle the second term. From the definition of $W^{c}$, if $V=\{1, \ldots, n\}$ and $2^{|V|}$ its power set we need only consider those sets $X \in 2^{|V|}$ with $|X| \leq(n / \mathrm{e})$,

$$
V_{s}:=\left\{(X, Y) \in 2^{|V|} \times 2^{|V|}: \max \{|X|,|Y|\} \leq n / \mathrm{e}\right\} .
$$

Before proceeding, we first need to establish an estimate on the random variable edge $(X, Y)$ when $(X, Y) \in$ $V_{s}$ :

Lemma A.6. For $(X, Y) \in V_{s}$ let edge $(X, Y)$ denote the random variable corresponding to the number of edges from an Erdös-Rényi random graph $\mathcal{G}(n, p)$ between $X$ and $Y$. Let $\mu(X, Y)=\mathbb{E}(\operatorname{edge}(X, Y))$ and $\mathcal{M}(X, Y):=\max \{|X|,|Y|\}$. Given any $\alpha>0$ let $k_{0}(X, Y)$ denote the unique solution to

$$
k_{0} \log \left(k_{0} / \mathrm{e}\right)=\frac{\alpha+4}{\mu} \mathcal{M} \log \frac{n \mathrm{e}}{\mathcal{M}} .
$$

Then

$$
\mathbb{P}\left(\max _{V_{s}}\left(\operatorname{edge}(X, Y)-k_{0}(X, Y) \mu(X, Y)\right)>0\right) \leq n^{-\alpha}
$$


Proof. From the union bound, it follows (through slight abuse of notation) that

$$
\begin{aligned}
& \mathbb{P}\left(\max _{V_{s}}\left(\operatorname{edge}(X, Y)-k_{0}(X, Y) \mu(X, Y)\right)>0\right) \leq \\
& \sum_{|X|=1}^{n / \mathrm{e}} \sum_{|Y|=1}^{n / \mathrm{e}} \sum_{X}^{\left(\begin{array}{c}
n \\
|X|
\end{array}\right)} \sum_{Y}^{\left(\begin{array}{c}
n \\
|Y|
\end{array}\right)} \mathbb{P}\left(\operatorname{edge}(X, Y)>k_{0}(X, Y) \mu(X, Y)\right)
\end{aligned}
$$

Using a one-sided concentration inequality (c.f. [26]) for sums of indicator variables,

$$
\mathbb{P}(\operatorname{edge}(X, Y)>k \mu(X, Y)) \leq \mathrm{e}^{-k \log (k / \mathrm{e}) \mu(X, Y)},
$$

which is valid for any $k>1$, shows that

$$
\mathbb{P}\left(\operatorname{edge}(X, Y)>k_{0}(X, Y) \mu(X, Y)\right) \leq \mathrm{e}^{-(\alpha+4) \mathcal{M} \log (n \mathrm{e} / \mathcal{M})}
$$

since $k_{0}>1$. It therefore follows that

$$
\begin{aligned}
& \sum_{|X|=1}^{n / \mathrm{e}} \sum_{|Y|=1}^{n / \mathrm{e}} \sum_{X}^{\left(\begin{array}{c}
n \\
(X \mid
\end{array}\right)} \sum_{Y}^{\left(\begin{array}{c}
n \\
|Y|
\end{array}\right)} \mathbb{P}\left(\operatorname{edge}(X, Y)>k_{0}(X, Y) \mu(X, Y)\right) \leq \sum_{|X|=1}^{n / \mathrm{e}} \sum_{|Y|=1}^{n / \mathrm{e}}\left(\begin{array}{c}
n \\
|X|
\end{array}\right)\left(\begin{array}{c}
n \\
|Y|
\end{array}\right) \mathrm{e}^{-(\alpha+4) \mathcal{M} \log (n \mathrm{e} / \mathcal{M})} \\
& \sum_{|X|=1}^{n / \mathrm{e}} \sum_{|Y|=1}^{n / \mathrm{e}} \exp \{|X| \log (n \mathrm{e} /|X|)+|Y| \log (n \mathrm{e} /|Y|)-(\alpha+4) \mathcal{M} \log (n \mathrm{e} / \mathcal{M})\} .
\end{aligned}
$$

The fact that $f(x):=x \log (n \mathrm{e} / x)$ is increasing for $1 \leq x \leq n$ then implies

$$
|X| \log (n \mathrm{e} /|X|)+|Y| \log (n \mathrm{e} /|Y|) \leq 2 \mathcal{M} \log (n \mathrm{e} / \mathcal{M}), \quad \mathcal{M} \log (n \mathrm{e} / \mathcal{M}) \geq 1+\log n .
$$

Combining this with the previous estimate yields

$$
\mathbb{P}\left(\max _{V_{s}}\left(\operatorname{edge}(X, Y)-k_{0}(X, Y) \mu(X, Y)\right)>0\right) \leq \sum_{|X|=1}^{n / \mathrm{e}} \sum_{|Y|=1}^{n / \mathrm{e}} n^{-(\alpha+2)} \leq n^{-\alpha}
$$

as desired.

The definition of $k_{0}(X, Y)$ and the fact $\mu(X, Y) \leq p|X||Y|$ then combine to yield the following corollary, which provides the basic estimate for the remaining contribution of $W^{c}$ to the sum.

Corollary A.7. With probability at least $1-n^{-\alpha}$, the estimate

$$
\operatorname{edge}(X, Y) \log \frac{\operatorname{edge}(X, Y)}{\operatorname{ep}|X||Y|} \leq(\alpha+4) \mathcal{M}(X, Y) \log \frac{n \mathrm{e}}{\mathcal{M}(X, Y)}
$$

holds for all $X, Y \in V_{s}$.

Estimating the second term in (55)

$$
\sum_{W^{c}} \operatorname{edge}\left(X_{i}, Y_{j}\right) \frac{2^{i} 2^{j}}{n}
$$

now proceeds by decomposing into the following cases:

$$
\begin{aligned}
\mathrm{I} & :=W^{c} \cap\left\{\operatorname{edge}\left(X_{i}, Y_{j}\right) \leq \mathrm{e}^{2} \sqrt{p / n}\left|X_{i}\right|\left|Y_{j}\right| 2^{i} 2^{j}\right\} \\
\mathrm{II}_{\mathrm{A}} & :=W^{c} \cap\left\{2^{i}>\sqrt{n p} 2^{j}\right\} \quad \mathrm{II}_{\mathrm{B}}:=W^{c} \cap\left\{2^{j}>\sqrt{n p} 2^{i}\right\} \\
\mathrm{III} & :=W^{c} \backslash\left(\mathrm{I} \cup \mathrm{II}_{\mathrm{A}} \cup \mathrm{II}_{\mathrm{B}}\right) .
\end{aligned}
$$


On I we have that

$$
\sum_{\mathrm{I}} \leq \mathrm{e}^{2} \sqrt{n p} \sum_{i, j} 2^{2 i}\left|X_{i}\right| 2^{2 j}\left|Y_{j}\right| n^{-2}=O(\sqrt{n p})
$$

due to $(56)$ as before. $O \mathrm{OI}_{\mathrm{A}}$ and $\mathrm{II}_{\mathrm{B}}$ the bounded degree property implies that edge $\left(X_{i}, Y_{j}\right) \leq c_{1} n p\left|X_{i}\right|$ and edge $\left(X_{i}, Y_{j}\right) \leq c_{1} n p\left|Y_{j}\right|$, respectively. Therefore

$$
\sum_{\mathrm{II}_{\mathrm{A}}} \leq c_{1} \sqrt{n p} \sum_{\mathrm{II}_{\mathrm{A}}} \frac{\left|X_{i}\right| 2^{2 i}}{n} \frac{2^{j} \sqrt{n p}}{2^{i}}=c_{1} \sqrt{n p} \sum_{i} \frac{\left|X_{i}\right| 2^{2 i}}{n} \sum_{j} \frac{2^{j} \sqrt{n p}}{2^{i}} \mathbf{1}_{\mathrm{II}_{\mathrm{A}}}(i, j) .
$$

For fixed $i$ let $j_{\max }(i)$ denote the largest $j$ so that $(i, j) \in \mathrm{II}_{\mathrm{A}}$. Then

$$
\sum_{j} \frac{2^{j} \sqrt{n p}}{2^{i}} \mathbf{1}_{\mathrm{II}_{\mathrm{A}}}(i, j) \leq \frac{2^{j_{\max }(i)} \sqrt{n p}}{2^{i}} \sum_{j=0}^{\infty} 2^{-j} \leq 2 \Rightarrow \sum_{\mathrm{II}_{\mathrm{A}}} \leq 2 c_{1} \sqrt{n p} \sum_{i} \frac{\left|X_{i}\right| 2^{2 i}}{n}=O(\sqrt{n p})
$$

due to (56) once again. By reversing the roles of $i$ and $j$, a similar argument shows

$$
\sum_{\mathrm{II}_{\mathrm{B}}}=O(\sqrt{n p})
$$

as well. For the remaining set III, let III $=\mathrm{III}^{i>j} \cup \mathrm{III}^{j>i}$ where the notation signifies $\left|Y_{j}\right| \geq\left|X_{i}\right|$ on $\mathrm{III}^{j>i}$ and vice-versa. We treat the first set and leave the second as an exercise since it follows analogously. Using the probabilistic estimate (60) as a guide, we decompose further into

$$
\begin{aligned}
& \operatorname{III}_{\mathrm{A}}^{j>i}:=\operatorname{III}^{j>i} \cap\left\{4 \log \frac{\operatorname{edge}\left(X_{i}, Y_{j}\right)}{\mathrm{e} p\left|X_{i}\right|\left|Y_{j}\right|} \geq \log \frac{n \mathrm{e}}{\left|Y_{j}\right|}\right\} \\
& \mathrm{III}_{\mathrm{B}}^{j>i}:=\mathrm{III}^{j>i} \cap\left\{4 \log \frac{\operatorname{edge}\left(X_{i}, Y_{j}\right)}{\mathrm{e}\left|X_{i}\right|\left|Y_{j}\right|}<\log \frac{n \mathrm{e}}{\left|Y_{j}\right|}\right\} \cap\left\{\frac{n e}{\left|Y_{j}\right|} \leq 2^{4 j}\right\} \\
& \operatorname{III}_{\mathrm{C}}^{j>i}:=\operatorname{III}^{j>i} \cap\left\{4 \log \frac{\operatorname{edge}\left(X_{i}, Y_{j}\right)}{\mathrm{e} p\left|X_{i}\right|\left|Y_{j}\right|}<\log \frac{n \mathrm{e}}{\left|Y_{j}\right|}\right\} \cap\left\{\frac{n e}{\left|Y_{j}\right|}>2^{4 j}\right\}
\end{aligned}
$$

For the first set the estimate (60) implies

$$
\sum_{\mathrm{III}_{\mathrm{A}}^{j>i}} \leq 4(\alpha+4) \sqrt{n p} \sum_{j} \frac{\left|Y_{j}\right| 2^{2 j}}{n} \sum_{i} \frac{2^{i}}{2^{j} \sqrt{n p}} \mathbf{1}_{\mathrm{III}_{\mathrm{A}}^{j>i}}(i, j)=O(\sqrt{n p})
$$

due to the fact that $2^{j} \sqrt{n p}>2^{i}$, the geometric series argument from (61) and the estimate (56). For the second set note that if $(i, j) \in \mathrm{III}$ then $(i, j) \notin \mathrm{I}$, so that the inequality

$$
\operatorname{edge}\left(X_{i}, Y_{j}\right)>\mathrm{e}^{2} \sqrt{p / n}\left|X_{i}\right|\left|Y_{j}\right| 2^{i} 2^{j}
$$

holds on III by definition. This combines with the definition of $\mathrm{III}_{\mathrm{B}}^{j>i}$ to imply that

$$
\mathrm{e} \leq \mathrm{e} \frac{2^{i} 2^{j}}{\sqrt{n p}} \leq \frac{\operatorname{edge}\left(X_{i}, Y_{j}\right)}{\mathrm{ep}\left|X_{i}\right|\left|Y_{j}\right|} \leq 2^{j}
$$

which shows that $1 \leq \log \frac{\operatorname{edge}\left(X_{i}, Y_{j}\right)}{\operatorname{ep}\left|X_{i}\right|\left|Y_{j}\right|}$ and that $2^{i}<\sqrt{n p}$ as well. The estimate (60) therefore implies

$$
\sum_{\mathrm{III}_{\mathrm{B}}^{j>i}} \leq 4(\alpha+4) \sum_{\mathrm{III}_{\mathrm{B}}^{j>i}} \frac{\left|Y_{j}\right| \log \left(2^{j}\right) 2^{i} 2^{j}}{n} \leq 4(\alpha+4) \sqrt{n p} \sum_{j} \frac{\left|Y_{j}\right| 2^{2 j}}{n} \sum_{i} \frac{2^{i}}{\sqrt{n p}} \mathbf{1}_{\mathrm{III}_{\mathrm{B}}^{j>i}}(i, j)=O(\sqrt{n p})
$$

due to the fact that $2^{i}>\sqrt{n p}$, the geometric series argument (61) and the estimate (56), exactly as before. On the final set the facts that

$$
\frac{n e}{\left|Y_{j}\right|}<\left(\frac{n e}{\left|Y_{j}\right| 2^{2 j}}\right)^{2}, \quad 4 \leq 4 \log \frac{\operatorname{edge}\left(X_{i}, Y_{j}\right)}{\operatorname{ep}\left|X_{i}\right|\left|Y_{j}\right|}<2 \log \frac{n e}{\left|Y_{j}\right| 2^{2 j}}<4 \log \frac{n e}{\left|Y_{j}\right| 2^{2 j}}
$$


imply that edge $\left(X_{i}, Y_{j}\right) \leq \mathrm{e}^{2} n p\left|X_{i}\right| 2^{-2 j}$, whence

$$
\sum_{\mathrm{III}_{\mathrm{C}}^{j>i}} \leq e^{2} \sqrt{n p} \sum_{i} \frac{\left|X_{i}\right| 2^{2 i}}{n} \sum_{j} \frac{\sqrt{n p}}{2^{i} 2^{j}} \mathbf{1}_{\mathrm{III}_{\mathrm{C}}^{j>i}}(i, j) .
$$

Arguing as before, the fact that $2^{i} 2^{j}>\sqrt{n p}$ gives that each sum over $j$ is bounded by two, so that applying (56) one final time demonstrates that the sum is $O(\sqrt{n p})$.

The following lemma summarizes the preceeding arguments:

Lemma A.8. Let $n p>c_{0} \log n$ for any $c_{0}>0$ and $\alpha$ denote an arbitrary positive constant. Then there exists a $c>0$ independent of $n$ so that with probability at least $1-n^{-\alpha}$ the estimate

$$
|H(\mathbf{x}, \mathbf{y})| \leq c \sqrt{n p}
$$

holds for all $(\mathbf{x}, \mathbf{y}) \in T_{0}^{\delta} \times T^{\delta}$.

Combining this with the estimate for $S(\mathbf{x}, \mathbf{y})$ and the reduction to the discrete set $T_{0}^{\delta} \times T^{\delta}$ yields the theorem.

\section{References}

[1] M. Aldana and C. Huepe. Phase transitions in self-driven many-particle systems and related nonequilibrium models: A network approach. Journal of Statistical Physics, 112(1):135-153, 2003.

[2] Eric L. Altschuler, Timothy J. Williams, Edward R. Ratner, Robert Tipton, Richard Stong, Farid Dowla, and Frederick Wooten. Possible global minimum lattice configurations for thomson's problem of charges on a sphere. Phys. Rev. Lett., 78(14):2681-2685, Apr 1997.

[3] Necdet Batir. Some new inequalities for gamma and polygamma functions. Journal of Inequalities in Pure and Applied Mathematics, 6(4):1-9, 2005.

[4] Silvia Boi, Vincenzo Capasso, and Daniela Morale. Modeling the aggregative behavior of ants of the species polyergus rufescens. Nonlinear Anal.: Real World Appl., 1:163-176, March 2000.

[5] J. Buhl, DJT Sumpter, ID Couzin, JJ Hale, E. Despland, ER Miller, and SJ Simpson. From disorder to order in marching locusts. Science, 312(5778):1402-1406, 2006.

[6] M. Cao, A.S. Morse, and B.D.O. Anderson. Reaching an agreement using delayed information. Decision and Control, 2006 45th IEEE Conference on, pages 3375-3380, Dec. 2006.

[7] Yao-Li Chuang, Y.R. Huang, M.R. D'Orsogna, and A.L. Bertozzi. Multi-vehicle flocking: Scalability of cooperative control algorithms using pairwise potentials. In Robotics and Automation, 2007 IEEE International Conference on, pages $2292-2299,2007$.

[8] Henry Cohn and Abhinav Kumar. Universally optimal distribution of points on spheres. J. Amer. Math. Soc., 20(1):99-148, 2007.

[9] Henry Cohn and Abhinav Kumar. Algorithmic design of self-assembling structures. P. Natl. Acad. Sci. USA, 106(24):9570-9575, 2009.

[10] Pierre Degond and Sebastien Motsch. Large scale dynamics of the persistent turning walker model of fish behavior. Journal of Statistical Physics, 131:989-1021, 2008. 10.1007/s10955-008-9529-8.

[11] Anna M. Delprato, Azadeh Samadani, A. Kudrolli, and L. S. Tsimring. Swarming ring patterns in bacterial colonies exposed to ultraviolet radiation. Phys. Rev. Lett., 87(15):158102, Sep 2001.

[12] Leah Edelstein-Keshet, James Watmough, and Daniel Grunbaum. Do travelling band solutions describe cohesive swarms? An investigation for migratory locusts. J. Math. Bio., 36:515-549, 1998. $10.1007 / \mathrm{s} 002850050112$. 
[13] U. Feige and E. Ofek. Spectral techniques applied to sparse random graphs. Random Structures 8 Algorithms, 27(2):251-275, 2005.

[14] E. Ferrante, AE Turgut, C. Huepe, M. Birattari, M. Dorigo, and T. Wenseleers. Explicit and implicit directional information transfer in collective motion. Artificial Life, 13:551-577, 2012.

[15] E. Ferrante, A.E. Turgut, C. Huepe, A. Stranieri, C. Pinciroli, and M. Dorigo. Self-organized flocking with a mobile robot swarm: a novel motion control method. Complete data, 2012.

[16] S. C. Glotzer and M. J. Solomon. Anisotropy of building blocks and their assembly into complex structures. Nature Materials, 6, 2007.

[17] S.C. Glotzer. Some assembly required. Science, 306(5695):419-420, 2004.

[18] M.F. Hagan and D. Chandler. Dynamic pathways for viral capsid assembly. Biophysical journal, 91(1):42-54, 2006.

[19] Yuko Hatano and Mehran Mesbahi. Agreement over random networks. Automatic Control, IEEE Transactions on, 50(11):1867-1872, 2005.

[20] Darryl D. Holm and Vakhtang Putkaradze. Formation of clumps and patches in self-aggregation of finite-size particles. Phys. D, 220(2):183-196, 2006.

[21] C. Huepe, G. Zschaler, A.L. Do, and T. Gross. Adaptive-network models of swarm dynamics. New Journal of Physics, 13(7):073022, 2011.

[22] J.N. Israelachvili. Intermolecular and surface forces: revised third edition. Academic press, 2011.

[23] Zhipu Jin and A.L. Bertozzi. Environmental boundary tracking and estimation using multiple autonomous vehicles. In Decision and Control, 2007 46th IEEE Conference on, pages 4918 -4923, 2007.

[24] Soummya Kar and José MF Moura. Sensor networks with random links: Topology design for distributed consensus. Signal Processing, IEEE Transactions on, 56(7):3315-3326, 2008.

[25] A. B. Kuijlaars and E. B. Saff. Asymptotics for minimal discrete energy on the sphere. Trans. Amer. Math. Soc., 350(2):523-538, 1998.

[26] Colin McDiarmid. Concentration. In Probabilistic methods for algorithmic discrete mathematics, pages 195-248. Springer, 1998.

[27] L. Moreau. Stability of continuous-time distributed consensus algorithms. Decision and Control, 2004. CDC. 43rd IEEE Conference on, 4:3998-4003 Vol.4, Dec. 2004.

[28] L. Moreau. Stability of multiagent systems with time-dependent communication links. Automatic Control, IEEE Transactions on, 50(2):169-182, Feb. 2005.

[29] Sebastien Motsch and Eitan Tadmor. A new model for self-organized dynamics and its flocking behavior. Journal of Statistical Physics, 144:923-947, 2011. 10.1007/s10955-011-0285-9.

[30] R. Olfati-Saber. Flocking for multi-agent dynamic systems: algorithms and theory. Automatic Control, IEEE Transactions on, 51(3):401-420, March 2006.

[31] R. Olfati-Saber and R.M. Murray. Consensus problems in networks of agents with switching topology and time-delays. Automatic Control, IEEE Transactions on, 49(9):1520-1533, Sept. 2004.

[32] F. W. J. Olver, D. W. Lozier, R. F. Boisvert, and C. W. Clark, editors. NIST Handbook of Mathematical Functions. Cambridge University Press, New York, NY, 2010.

[33] A. Onufriev, D. Bashford, and D.A. Case. Exploring protein native states and large-scale conformational changes with a modified generalized born model. Proteins: Structure, Function, and Bioinformatics, $55(2): 383-394,2004$. 
[34] A. Pérez-Garrido, M. J. W. Dodgson, and M. A. Moore. Influence of dislocations in thomson's problem. Phys. Rev. B, 56(7):3640-3643, Aug 1997.

[35] M. Porfiri, D.J. Stilwell, EM Bollt, and JD Skufca. Stochastic synchronization over a moving neighborhood network. In American Control Conference, 2007. ACC'07, pages 1413-1418. IEEE, 2007.

[36] Maurizio Porfiri and Daniel J Stilwell. Consensus seeking over random weighted directed graphs. $A u-$ tomatic Control, IEEE Transactions on, 52(9):1767-1773, 2007.

[37] Maurizio Porfiri, Daniel J Stilwell, and Erik M Bollt. Synchronization in random weighted directed networks. Circuits and Systems I: Regular Papers, IEEE Transactions on, 55(10):3170-3177, 2008.

[38] W. Ren and E. M. Atkin. Distributed multi-vehicle coordinated control via local information exchange. International Journal of Robust and Nonlinear Control, 17(10-11):1002-1033, 2007.

[39] W. Ren and R. Beard. Distributed Consensus in Multi-vehicle Cooperative Control:Theory and Applications. Communications and Control Engineering. Springer, London, 2008.

[40] W. Ren, K. Moore, and Y. Chen. High-order and model reference consensus algorithms in cooperative control of multi-vehicle systems. ASME J. Dyn. Sys. Meas. Control, 129(5):678-688, 2007.

[41] Wei Ren and R.W. Beard. Consensus seeking in multiagent systems under dynamically changing interaction topologies. Automatic Control, IEEE Transactions on, 50(5):655-661, May 2005.

[42] G. Sigalov, A. Fenley, and A. Onufriev. Analytical electrostatics for biomolecules: Beyond the generalized born approximation. The Journal of chemical physics, 124:124902, 2006.

[43] JD Skufca and EM Bollt. Communication and synchronization in, disconnected networks with dynamic topology: moving neighborhood networks. Mathematical biosciences and engineering: MBE, 1(2):347, 2004.

[44] J. J. Thomson. On the structure of the atom. Philosophical Magazine, 7(39):237-265, 1904.

[45] Lev Tsimring, Herbert Levine, Igor Aranson, Eshel Ben-Jacob, Inon Cohen, Ofer Shochet, and William N. Reynolds. Aggregation patterns in stressed bacteria. Phys. Rev. Lett., 75(9):1859-1862, Aug 1995.

[46] Carel J. van Oss. Interfacial Forces in Aqueous Media. Taylor \& Francis Group, Florida, 2nd edition, 2006.

[47] CJ Van Oss, RF Giese, and P.M. Costanzo. Dlvo and non-dlvo interactions in hectorite. Clays and Clay Minerals, 38(2):151-159, 1990.

[48] Tamás Vicsek, András Czirók, Eshel Ben-Jacob, Inon Cohen, and Ofer Shochet. Novel type of phase transition in a system of self-driven particles. Phys. Rev. Lett., 75:1226-1229, Aug 1995.

[49] James von Brecht, Theodore Kolokolnikov, Andrea L Bertozzi, and Hui Sun. Swarming on random graphs. Journal of Statistical Physics, pages 1-24, 2013.

[50] McKay Hayley Wales, David J. and Eric L. Altschuler. Defect motifs for spherical topologies. Phys. Rev. B, 79(22):224115, Jun 2009.

[51] Wen Yang, A.L. Bertozzi, and Xiaofan Wang. Stability of a second order consensus algorithm with time delay. In Decision and Control, 2008. CDC 2008. 47th IEEE Conference on, pages 2926 -2931, 2008.

[52] R. Zandi, D. Reguera, R.F. Bruinsma, W.M. Gelbart, and J. Rudnick. Origin of icosahedral symmetry in viruses. P. Natl. Acad. Sci. USA, 101(44):15556-60, 2004.

[53] Z. Zhang and S. C. Glotzer. Self-Assembly of Patchy Particles. Nano Letters, 4(8), 2004. 